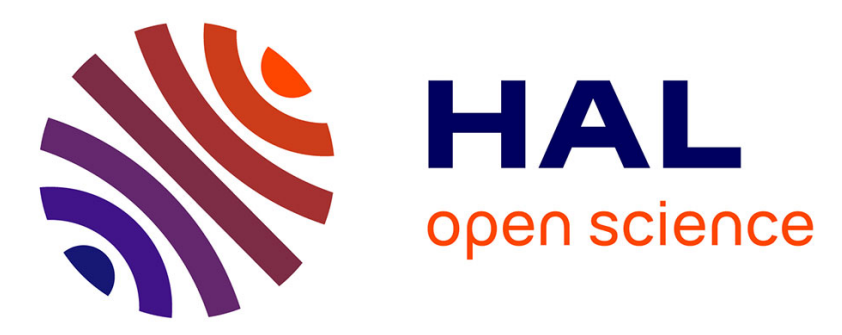

\title{
A stochastic projection-based hyperreduced order model for model-form uncertainties in vibration analysis
}

Charbel Farhat, Adrien Bos, Radek Tezaur, Todd Chapman, Philip Avery, Christian Soize

\section{- To cite this version:}

Charbel Farhat, Adrien Bos, Radek Tezaur, Todd Chapman, Philip Avery, et al.. A stochastic projection-based hyperreduced order model for model-form uncertainties in vibration analysis. 2018 AIAA Non-Deterministic Approaches Conference, AIAA SciTech Forum 2018, AIAA, Jan 2018, Kissimmee, Florida, United States. pp.1-20, 10.2514/6.2018-1410 . hal-01685246

\section{HAL Id: hal-01685246 https://hal.science/hal-01685246}

Submitted on 16 Jan 2018

HAL is a multi-disciplinary open access archive for the deposit and dissemination of scientific research documents, whether they are published or not. The documents may come from teaching and research institutions in France or abroad, or from public or private research centers.
L'archive ouverte pluridisciplinaire HAL, est destinée au dépôt et à la diffusion de documents scientifiques de niveau recherche, publiés ou non, émanant des établissements d'enseignement et de recherche français ou étrangers, des laboratoires publics ou privés. 


\title{
A Stochastic Projection-Based Hyperreduced Order Model for Model-Form Uncertainties in Vibration Analysis
}

\author{
Charbel Farhat*, Adrien Bos ${ }^{\dagger}$, Radek Tezaur ${ }^{\ddagger}$, Todd Chapman ${ }^{\S}$ \\ Stanford University, Stanford, CA 94305, USA \\ Philip Avery ${ }^{\text {II }}$ \\ US Army Research Laboratory, Adelphi, MD \\ and Stanford University, Stanford, CA 94305, USA \\ and Christian Soize" \\ Université Paris-Est, 77454 Marne-la-Vallee, France
}

\begin{abstract}
A feasible, nonparametric, probabilistic approach for quantifying model-form uncertainties associated with a High-Dimensional computational Model (HDM) and/or a corresponding Hyperreduced Projection-based Reduced-Order Model (HPROM) designed for the solution of generalized eigenvalue problems arising in vibration analysis, is presented. It is based on the construction of a Stochastic HPROM (SHPROM) associated with the HDM and its HPROM using three innovative ideas: the substitution of the deterministic Reduced-Order Basis (ROB) with a Stochastic counterpart (SROB) that features a reduced number of hyperparameters; the construction of this SROB on a compact Stiefel manifold in order to guarantee the linear independence of its column vectors and the satisfaction of any applicable constraints; and the formulation and solution of a reduced-order inverse statistical problem to determine the hyperparameters so that the mean value and statistical fluctuations of the eigenvalues predicted in real time using the SHPROM match target values obtained from available data. If the data are experimental data, the proposed approach models and quantifies the model-form uncertainties associated with the HDM, while accounting for the modeling errors introduced by model reduction. If on the other hand the data are high-dimensional numerical data, the proposed approach models and quantifies the model-form uncertainties associated with the HPROM. Consequently, the proposed nonparametric, probabilistic approach for modeling and quantifying model-form uncertainties can also be interpreted as an effective means for extracting fundamental information or knowledge from data that is not captured by a deterministic computational model, and incorporating it in this model. Its potential for quantifying model-form uncertainties in eigenvalue computations is demonstrated for what-if? vibration analysis scenarios associated with shape changes for a jet engine nozzle.
\end{abstract}

\section{Introduction}

In computational modeling, one can distinguish at least between two sources of uncertainty: parameter uncertainty, and model-form uncertainty. Parameter uncertainty is associated with those parameters of the mathematical model whose exact values are unknown to or cannot be controlled by experimentalists, or whose values cannot be exactly inferred by statistical methods [1]. Model-form uncertainties can result either from the lack of knowledge of the true physics underlying the problem of interest, or the omission or truncation of modeling details, for reasons ranging from the lack of available information to the inability to discern in some circumstances between important and unimportant

*Vivian Church Hoff Professor of Aircraft Structures, Department of Aeronautics and Astronautics, Durand Building, Room 257, Stanford University, Stanford, CA 94305-3035. AIAA Fellow. Email: cfarhat@stanford.edu .

${ }^{\dagger}$ Graduate Student, Department of Aeronautics and Astronautics, Durand Building Room 028, Stanford University, Stanford, CA 94305-3035. Email: abos@stanford.edu.

${ }^{\ddagger}$ Senior Research Engineer, Department of Aeronautics and Astronautics, Durand Building, Room 214, Stanford University, Stanford, CA 94305-3035. Email: rtezaur@stanford.edu.

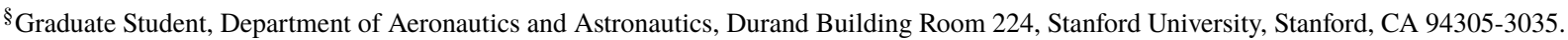
Email: tac688@stanford.edu.

IIUS Army Research Laboratory, Adelphi, MD, USA and Department of Aeronautics and Astronautics, Durand Building, Room 214, Stanford University, Stanford, CA 94305-3035. Email: pavery@stanford.edu.

" Professor, Laboratoire Modélisation et Simulation Multi Echelle, MSME UMR 8208 CNRS, Université Paris-Est, 5 bd Descartes, 77454 Marne-la-Vallee, France. E-mail: christian. soize@univ-paris-est.fr. 
modeling details. Such uncertainties affect the ability of even a High-Dimensional Model (HDM) to deliver predictive results - that is, results that match reasonably well their experimental counterparts (which themselves can also be tainted by other types of uncertainties). Examples in computational structural dynamics include constitutive, multiscale, friction, homogenization, and free-play modeling errors. In the literature, the modeling, quantification, and propagation of parameter uncertainty has dominated the scene. By comparison, model-form uncertainty has been far less studied: therefore, its modeling and quantification are still in their infancies. This, despite the fact that once a model must be considered for any purpose, model-form uncertainties are unavoidable.

Indeed, many if not most of the approaches developed so far for Uncertainty Quantification (UQ) are based on parametric probabilistic methods, and prior and a posterior stochastic models of uncertain model parameters pertaining, for example, to geometry, boundary conditions, and material properties. Such approaches are particularly well developed for modeling model parameter uncertainty, at least for a small number of parameters. Their computational feasibility has been demonstrated for relatively small-size HDMs. For larger-scale HDMs, the underlying Monte Carlo simulations, or other type of stochastic computations, become so computationally intensive that model reduction becomes unavoidable. Unfortunately, model reduction introduces in the process its own model-form uncertainties which parametric probabilistic methods for UQ cannot take into account.

For example, Projection-based Model Order Reduction (PMOR) is one approach to achieve model order reduction that is grounded in mathematics. It is also the most popular and perhaps the most rigorous approach for reducing the dimension of an HDM. It achieves this objective by projecting the HDM onto a subspace of lower dimension represented by a Reduced-Order Basis (ROB). This projection requires first constructing this ROB, then approximating the solution in the lower-dimensional subspace described by this ROB, and finally projecting some form of the governing equations associated with the HDM onto the same ROB or a related one. The outcome of PMOR is a Projection-based Reduced-Order Model (PROM). This reduced-order model distinguishes itself from simplified models that are also often called reduced-order models as follows: if the ROB is carefully constructed, the PROM can maintain most of the fidelity of the HDM, which a simplified model can rarely do. Unfortunately, a PROM inherits by construction any form of uncertainty in its underlying HDM. It also inherits the model-form uncertainties associated with the construction of the ROB onto which the HDM is projected to obtain this PROM. These uncertainties include at least:

- The uncertainty associated with finite sampling in the design of experiments type of procedure performed offline (and usually referred to as training or learning) to construct a global ROB whose domain of validity covers a large part of the design space.

- That associated with the model truncation errors due to the truncation of the subspace of approximation underlying such a ROB via a data compression method such as, for example, SVD (Singular Value Decomposition) or POD (Proper Orthogonal Decomposition).

- The uncertainty arising from the approximation errors introduced by hyperreduction to achieve computational feasibility.

Hence, a PROM that is used to accelerate stochastic computations propagates in these computations the above model-form uncertainties which cannot be accounted for by parametric probabilistic methods for UQ. Considering that such methods are often designed for modeling model parameter uncertainty, it follows that at least in this case, modeling and quantifying model-form uncertainty is a pre-requisite for studying parameter uncertainty.

Even though the issues and solution approaches discussed in this paper are very broad, the focus of this work is on vibration analysis, and more specifically, on the associated generalized EigenValue Problem (EVP). For structural dynamics analysis in general, nonparametric probabilistic approaches for modeling uncertainties induced by general modeling errors been have been developed in [2-4], albeit for linear problems and a limited class of geometrically nonlinear structural analysis problems. More recently, a new nonparametric probabilistic approach based on the construction of a Stochastic PROM (SPROM) was recently introduced in [5] for addressing specifically model-form uncertainties, and the broader context of arbitrarily nonlinear structural dynamics problems. In [6], its scope was extended to generalized eigencomputations, as these are important in many fields including vibration analysis. The resulting method was also successfully demonstrated for a jet engine application for which a small-scale Finite Element (FE) HDM and experimental data are available. However, even for medium-scale HDMs, this extension to the generalized EVP of the nonparametric, probabilistic approach proposed first in [5] for modeling and quantifying model-form uncertainties in nonlinear structural dynamics applications is practically infeasible. This paper explains the reasons why this is the case. It also remedies this issue by redesigning the original model-form UQ method originally proposed in [5] around the concept of a Stochastic Hyperreduced PROM (SHPROM) rather than an SPROM. It also demonstrates for the first time the ability of this method to operate outside the training parameter points of its Hyperreduced PROM (HPROM), by illustrating its application to what-if? vibration analysis scenarios associated with shape changes of a jet 
engine nozzle.

For this purpose, the focus of this paper is specifically set on the discrete, generalized EVP associated with a given FE structural dynamics model, and on the modeling and quantification of model-form uncertainties in such a model for which an HPROM can be constructed.

\section{Projection-based dimensional reduction of parametric generalized eigenvalue problems}

Here and throughout the remainder of this paper, the words "parameter", "parametric", and "parameterized" do not refer nor relate to parametric probabilistic approaches for UQ, but to a parameterized (or parametric) computational model such as an HDM, PROM, HPROM, SPROM, or SHPROM - that is, a computational model that depends on a vector of deterministic parameters that are varied for the purpose of performing parametric studies. Such studies are typically computationally intensive. When the HDM and/or the parameter domain have very large dimensions, they may be even practically infeasible, unless they are performed using a reduced-order model such as a PROM or an HPROM. As a matter of fact, parametric studies, which include stochastic studies as a particular case, are one of the driving applications of model reduction.

\section{A. High-dimensional form}

A parameterized, discrete, generalized EVP associated with a structural dynamics FE HDM of dimension $N$ can be written as

$$
K(\varrho) u(\varrho)=\omega(\varrho)^{2} M(\varrho) u(\varrho)
$$

where $\varrho \in \Omega_{\varrho} \subset \mathbb{R}^{m_{\varrho}}$ is a vector of deterministic, physical or nonphysical model parameters, $\Omega_{\varrho}$ is a parameter domain of interest and $m_{\varrho}$ is its dimension, $M(\varrho) \in \mathbb{R}^{N \times N}$ and $K(\varrho) \in \mathbb{R}^{N \times N}$ are the FE mass and stiffness matrices, respectively, and the notation $\bullet(\varrho)$ emphasizes the dependence of the quantity $\bullet$ on $\varrho$. Occasionally, this dependence is not explicitly highlighted in the remainder of this paper for the sake of brevity. The vector $u(\varrho) \in \mathbb{R}^{N}$ denotes the semi-discrete amplitude of an assumed harmonic vibration of the structure (also called the eigenmode), $\omega(\varrho)=2 \pi f(\varrho)$ is the corresponding circular frequency, and $f$ is the ordinary frequency. It is assumed that the displacement boundary conditions are incorporated into the above governing equation, in which case the dimension $N$ of the given FE HDM is equal to its number of unconstrained unknowns.

For large-scale FE models (large values of $N$ ), and in particular when the problem is parameterized by many parameters (large values of $m_{\varrho}$ ) and/or over a large parameter domain $\Omega_{\varrho}$, the solution of the generalized EVP (II.1) becomes computationally very intensive. Practical interest is usually focused on a subset of $m_{u}$ eigenvalues and eigenvectors at the lower end of the frequency spectrum: this is because the high eigenvalues and the associated eigenvectors are often unresolved by the mesh underlying the HDM, and therefore non-physical mesh frequencies and corresponding eigenvectors are captured instead. Even in this case, reducing the dimension of the problem (III.1) before solving it in the parameter domain of interest is attractive for many applications. However, the reduction of this problem is considered here primarily for the purpose of modeling and quantifying the model-form uncertainties affecting $M(\varrho)$, $K(\varrho)$, and the $m_{u}$ pairs of eigensolutions $\left(\omega_{i}(\varrho)^{2}, u_{i}\right), i=1, \cdots, m_{u}<N$. Indeed, it will be shown that in this case, dimension reduction may become interesting if not necessary even for relatively small values of $N$.

The natural frequencies $f_{i}(\varrho)=\omega_{i}(\varrho) / 2 \pi, i=1, \cdots, m_{u}<N$, are defined in this paper as the Quantities of Interest (QoIs) for problem (II.1).

\section{B. Projection-based dimensional reduction using a global reduced-order basis}

In order to accelerate the solution of the generalized EVP (II.1) over the parameter domain $\Omega_{\varrho} \subset \mathbb{R}^{m_{\varrho}}$, a PROM is considered here for this application. This PROM is constructed by projecting the governing equation (II.1) onto a small subspace spanned by a ROB, which leads to an approximation of this EVP of lower dimension. To achieve accuracy over the entire parameter domain, a global ROB is built from local subspaces constructed at a few sampled parameter points in $\Omega_{\varrho}$. Details about the implied sampling procedure are given in the following section. Here, the focus is set instead on the construction of a global ROB - that is, a ROB that leads to accurate approximations in a large part of, if not the entire domain $\Omega_{\varrho}$ - given an $n_{\varrho}$-point sampling $\left\{\varrho_{i}\right\}_{i=1}^{i=n_{\varrho}}$ of this parameter domain.

In the context of structural dynamics problems, a modal basis consisting of eigenvectors $u_{i}(\varrho), i=1, \cdots, m_{u}<N$ obtained from the numerical solution at one parameter point $\varrho_{\star} \in \Omega_{\varrho}$ of problem (III.1) is used as a ROB to reduce the dimension of this problem at this parameter point. However, such a ROB cannot be considered in this work since the main interest here is in modeling and quantifying the effect of model-form uncertainties on the computation of 
the eigenpairs $\left(\omega_{i}(\varrho)^{2}, u_{i}(\varrho)\right), i=1, \cdots, m_{u}$. Hence, an alternative ROB $V \in \mathbb{R}^{N \times n}$, where $m_{u} \leq n \ll N$, is built in this work by applying the Proper Orthogonal Decomposition (POD) method and the method of snapshots [7] to the following time domain equivalent of problem (II.1)

$$
\begin{aligned}
M(\varrho) \ddot{u}(\varrho, t)+K(\varrho) u(\varrho, t) & =f^{\mathrm{ext}}(\varrho, t) \\
u(\varrho, 0) & =u_{0}(\varrho) \\
\dot{u}(\varrho, 0) & =v_{0}(\varrho)
\end{aligned}
$$

where $t$ denotes time, a dot designates a time derivative, and $f^{\text {ext }}(\varrho, t), u_{0}(\varrho)$ and $v_{0}(\varrho)$ denote the semi-discrete, time-dependent, external force vector and displacement and velocity initial conditions, respectively. The external force vector and the initial conditions are chosen so as to generate a broadband excitation of the modeled structural dynamics system.

Specifically, the semi-discrete initial-value problem III.2 is solved for each sampled parameter point $\varrho \in\left\{\varrho_{i}\right\}_{i=1}^{i=n_{\varrho}}$ using a preferred time-integrator. The computed solutions are collected at a chosen time-interval frequency into a global snapshot matrix $X \in \mathbb{R}^{N \times n_{s}}$, where $n_{s}$ denotes the total number of collected solution snapshots. Then, the snapshot matrix $X$ is compressed using, for example, the SVD method, in which case the first $n$ left singular vectors associated with the $n$ dominant singular values are selected to form the global ROB $V \in \mathbb{R}^{N \times n}$. Denoting these $n$ singular values by $\sigma_{1}, \sigma_{2}, \cdots, \sigma_{n}$ and ordering them so that $\sigma_{1} \leq \sigma_{2} \leq \cdots \leq \sigma_{n}$, the accuracy of the approximation of $X$ by $V$ measured in the Frobenius norm is controlled by the ratio

$$
r_{\sigma}=\frac{\sum_{i=1}^{n} \sigma_{i}^{2}}{\sum_{i=1}^{n_{s}} \sigma_{i}^{2}}
$$

Thus, this ratio can be used to determine the appropriate dimension $n$ of the ROB by specifying an "energy" threshold and finding the smallest value of $n$ for which the above ratio exceeds this threshold.

For many structural dynamics applications, it is advantageous to choose an $M$-orthonormal ROB $V$ [8] - that is, a ROB $V$ which satisfies $V^{T} M V=I_{n}$, where $I_{n}$ denotes the identity matrix of dimension $n$. However for a parametric application, $M$ may vary from one parameter point of the parameter domain to another. For this reason, the standard orthonormality condition $V^{T} V=I_{n}$, which is satisfied by the ROB $V$ delivered by the standard SVD applied to the compression of the matrix of snapshots $X$, is adopted here.

Next, the generalized EVP (II.1) is projected onto the subspace spanned by the columns of $V$ using a Galerkin projection. To this end, a generalized eigenvector is approximated as

$$
u \approx V u_{r}
$$

where $u_{r}$ denotes the reduced vector of generalized coordinates, and substituted into (II.1). The resulting equation is premultiplied by $V^{T}$, where the superscript $T$ denotes here and throughout the remainder of this paper the transpose operation. This yields the following PROM for the discrete, generalized EVP (II.1)

$$
\begin{array}{rrr}
K_{r}(\varrho) u_{r}(\varrho) & = & \varpi^{2}(\varrho) M_{r}(\varrho) u_{r}(\varrho) \\
& \text { where } \\
K_{r}(\varrho)=V^{T} K(\varrho) V \quad & \text { and } & M_{r}(\varrho)=V^{T} M(\varrho) V
\end{array}
$$

The notation $\varpi$ is introduced above to differentiate the circular frequencies governed by the PROM (II.5) from their counterparts governed by the HDM (II.1). It is noted that the problem (II.5] is of size $n \ll N$ and therefore can be solved in real time. It is also noted that if the ROB $V$ accurately represents the dynamic behavior of the FE HDM, the eigensolutions of the problem (II.5)

$$
\left(\varpi_{i}^{2}, V u_{r_{i}}\right), \quad i=1, \cdots, m_{u} \leq n \ll N
$$

can be expected to be accurate approximations of the eigensolutions of the problem (II.1).

\section{Parameter sampling and construction of the global reduced-order basis}

A PROM that is built at a single parameter point $\varrho_{\star}$ in the parameter domain $\Omega_{\varrho}$ is expected to perform well at this parameter point, and possibly in its immediate neighborhood. In order to build a PROM that performs well however in a 
large part of, if not the entire parameter domain $\Omega_{\varrho} \subset \mathbb{R}^{m_{\varrho}}$, an approach that constructs a global ROB (and then the corresponding PROM by projection onto this ROB) by sampling many parameter points in the parameter domain is often adopted. In general, it is difficult, if not impossible, to determine a priori the best parameter points for this purpose. Thus, a progressive, iterative approach usually referred to as the greedy [9, 10] sampling procedure or algorithm, is adopted instead. While this sampling algorithm is in general sub-optimal (in the sense of minimizing the number of parameter points to be sampled in $\Omega_{\varrho}$ or the dimension of the resulting global ROB $V$ ), it is easy to implement. It has also the merit of converting the difficult problem of finding the optimal parameter points $\varrho_{j} \in \Omega_{\varrho}$ into an easier one namely, finding the next point $\varrho_{j+1}$ that is optimal in some sense with respect to the present state of the list of sampled parameter points.

The greedy sampling algorithm has two principal ingredients:

1) A pre-sampling scheme that is used to find candidates for the next parameter point to be sampled.

2) An error indicator that is exploited to discriminate between the aforementioned candidate parameter points and to monitor the convergence of the progressively constructed global PROM.

At each iteration of this algorithm, candidate parameter points are pre-sampled in $\Omega_{\varrho}$. The nature of this pre-sampling depends on the application. For a parameter domain of small dimension, a uniform pre-sampling is affordable. For a parameter domain of a large dimension, sparse and random pre-samplings methods such as the Latin hypercube sampling method are more appropriate. Once the candidate parameter points have been determined, the error indicator is applied at each of these points and the candidate parameter point where the indicated error is the largest is sampled. If the cost of the offline phase is of no concern, the true error can be computed and used as the perfect indicator. However, this is seldom the case. For this reason, a computationally inexpensive and yet accurate error indicator such as the residual of the governing equation - or some quantity derived from it - is often adopted instead to discriminate at each iteration among the pre-sampled candidate parameter points.

Let $\mathrm{PROM}^{(k)}$ denote the PROM associated with the global ROB $V$ obtained after $k$ iterations of the greedy sampling algorithm, $\mathcal{S}^{(k)}=\left\{\varrho_{j}^{(k)}\right\}_{j=1}^{j=\varrho_{\varrho^{(k)}}}$, where $\left.\varrho_{j}^{(k)} \in \Omega_{\varrho}\right\}$ denote the set of candidate parameter points pre-sampled at iteration $k$, and $E R\left(\mathrm{PROM}, \rho_{\star}\right) \in \mathbb{R}$ denote the value of the error indicator for a given PROM at the parameter point $\varrho_{\star}$. Using these definitions and a specified tolerance $\epsilon$, the basic version of the greedy sampling algorithm which builds a global PROM that is in principle accurate (up to the tolerance $\epsilon$ ) in a large part of the parameter domain $\Omega_{\varrho}$ can be summarized as follows:

1) Initialize $k:=1$ and randomly sample the first parameter point $\varrho_{1}$.

2) Build $\operatorname{PROM}^{(k)}$ using the set of sampled parameter points $\left\{\varrho_{j}\right\}_{i=1}^{i=k}$.

3) Pre-sample a set of candidate parameter points $\mathcal{S}^{(k)}$

4) For each pre-sampled candidate parameter point $\varrho_{j}^{(k)} \in \mathcal{S}^{(k)}$, compute $\left|E R\left(\mathrm{PROM}^{(k)}, \varrho_{j}^{(k)}\right)\right|$

5) Sample the parameter point in $\mathcal{S}^{(k)}$ where the error indicator is maximized $\varrho_{k+1} \mid \varrho_{k+1} \in \mathcal{S}^{(k)}$ and $\forall \varrho_{j}^{(k)} \in \mathcal{S}^{(k)},\left|E R\left(\mathrm{PROM}^{(k)}, \varrho_{j}^{(k)}\right)\right| \leq\left|E R\left(\mathrm{PROM}^{(k)}, \varrho_{k+1}\right)\right|$

6) If $\left|E R\left(\mathrm{PROM}^{(k)}, \varrho_{k+1}\right)\right|<\epsilon$, then exit; else set $k:=k+1$ and go to 2 .

For the application considered in this paper, Step 2 of the above algorithm includes - as discussed in the previous section - solving numerically (III.2) to collect a set of snapshots at the sampled parameter point $\varrho_{k}$, updating the snapshot matrix $X$ by concatenating its content with these newly collected solution snapshots, and compressing the updated matrix $X$ using SVD and the truncation criterion (III.3) to update the global ROB $V$ and its dimension. Step 2 of the above algorithm also incorporates a hyperreduction approximation that is described in Section IV] This approximation transforms the updated PROM into an HPROM whose construction has a computational complexity that scales only with the small dimension $n$ of the associated PROM, which significantly accelerates the execution of the stochastic computations detailed in Section [III]

It is also noted that Step 3 has a few variants, as it can be implemented using: the same set of candidate parameter points throughout the iterations of the greedy sampling algorithm, as in the uniform sampling approach; or a random set of candidates generated either at the beginning of the algorithm, or at the beginning of every iteration of the algorithm.

For each eigenpair $\left(\omega_{i}(\varrho)^{2}, u_{i}(\varrho)\right)$ that is a solution of the generalized EVP (II.1), the residual associated with its approximation by the eigenpair $\left(\varpi_{i}^{2}, V u_{r_{i}}\right)$ that is a solution of the PROM II.5) can be written as

$$
r_{i}=\left(K-\varpi_{i}^{2} M\right) V u_{r_{i}}=\left(K-\omega_{i}^{2} M\right)\left(V u_{r_{i}}-u_{i}\right)+\left(\omega_{i}^{2}-\varpi_{i}^{2}\right) M V u_{r_{i}}
$$

This expression of $r_{i}$ indicates, as expected, that two errors contribute to the residual $r_{i}$ : one associated with the 
approximation of the eigenvector; and another error associated with the approximation of the eigenvalue. For this reason, and because the objective of the greedy sampling algorithm is to enable the construction of a global PROM that delivers in this case accurate approximations of the first $m_{u}$ eigenvalues of (II.1) in a large part of the parameter domain - specifically, the subdomain of $\Omega_{\varrho}$ defined by the envelope of the set of $n_{\varrho}$ sampled parameter points $\left\{\varrho_{i}\right\}_{i=1}^{i=n_{\varrho}}-$ the following, residual-based, family of error indicators was first considered in this work

$$
r_{i}=\sum_{i=1}^{m_{u}} \frac{\left\|\left(K-\varpi_{i}^{2} M\right) V u_{r_{i}}\right\|_{2}}{\operatorname{op}\left(\left\|K V u_{r_{i}}\right\|_{2}, \varpi_{i}^{2}\left\|M V u_{r_{i}}\right\|_{2}\right)}
$$

where the operator op can be either the sum, maximum, minimum, first operand, or second operand. Practical experience revealed that for all aforementioned operators op, the family of error indicators (II.7) leads to a reasonable performance of the greedy sampling algorithm. However, practical experience also revealed that the following variant of the above family of error indicators maintains a relatively small and constant discrepancy factor with respect to the true error

$$
r_{i}=\sum_{i=1}^{m_{u}} \frac{\left|u_{r_{i}}^{T} V^{T} M^{T}\left(K-\varpi_{i}^{2} M\right) V u_{r_{i}}\right|}{\varpi_{i}^{2}\left\|M V u_{r_{i}}\right\|_{2}^{2}}
$$

It is worthwhile noting that whereas neither of the error indicators (II.7) and (II.8) provides a sharp upper or lower bound on the true error, both of them can be calibrated by the true error computed at the parameter point where the error indicator is maximized. Such a calibration makes the error indicator bear a closer relationship to the true error in terms of scale, which in turn makes the choice of convergence tolerance $\epsilon$ more meaningful for the user.

\section{Data-driven construction of a stochastic projection-based reduced-order model}

\section{A. Data-driven model adaptation}

In [5], an innovative, nonparametric, probabilistic approach based on PMOR was developed to model and quantify model-form uncertainties in a given deterministic computational model. Simply put, the main idea behind this method can be described as the following six-fold idea:

- Recognize that data, whether experimental or numerical and high-dimensional, may contain fundamental information or knowledge that is not captured by the given deterministic computational model (which is similar to recognizing that once a model must be considered for any purpose, model-form uncertainties are unavoidable).

- Adapt the subspace in which the solution of the computational model is searched for rather than the model parameters in order to discover some form of this information or knowledge, which requires parameterizing this subspace.

- Parameterize this subspace by randomizing its representative basis using hyperparameters, which transforms the deterministic computational model into a hyperparametric stochastic one.

- Determine these hyperparameters by constructing and solving an inverse statistical problem designed to minimize the discrepancies between the mean value and statistical fluctuations of the QoIs predicted using the hyperparametric stochastic model and target values obtained from the available data.

- Implement all of the above ideas into a surrogate, lower-dimensional model if the given deterministic model is an HDM in order to make them computationally feasible, and choose as surrogate model a PROM because it can be built to preserve the needed fidelity.

- Recognize that the above steps amount to extracting from the available data the information missing in the deterministic computational model and infusing it into a stochastic, lower-dimensional counterpart, which is why these steps are described here as a data-driven model adaptation.

This six-fold idea addresses both types of model-form uncertainties outlined in Section $\mathbb{I}$ those inherent to the HDM, because they are inherited by its PROM surrogate; and the modeling errors introduced by the model reduction process used to construct the PROM. If experimental data are not available, this six-fold idea leads to the modeling and quantification of the modeling errors due to model reduction only. Otherwise, it leads to a nonparametric probabilistic approach for performing model-form UQ of the given HDM, while accounting for all modeling errors introduced during model reduction.

In [5], it was proposed to randomize the ROB $V$ in order to transform the deterministic solution of the problem of interest into a stochastic one that can adapt to the available data so that it can learn from it. To this effect, it is noted here 
that throughout the remainder of this paper, the bold font is used to designate a stochastic quantity. For example, $\mathbf{V}$ denotes the Stochastic ROB (SROB) associated with the deterministic ROB $V$.

However, the randomization of $V$ cannot be performed arbitrarily, because $\mathbf{V}$ must remain an admissible basis of global modes. Hence, in all events, its columns must remain linearly independent. A simple, albeit stronger approach for enforcing almost surely the linear independency of the columns of the SROB $\mathbf{V}$ is to preserve almost surely the orthonormality property satisfied by its deterministic counterpart $V$. Therefore, if $V \in \mathbb{R}^{N \times n}$ is constructed as stated earlier to satisfy $V^{T} V=I_{n}$, the SROB $\mathbf{V}$ is constructed so that:

- $\mathbf{V} \in \mathbb{R}^{N \times n}$ is a random matrix whose probability distribution is constructed using the Maximum Entropy principle of Information Theory and a stochastic representation of random fields.

- The support of this probability distribution is the subset of real $N \times n$ matrices satisfying the constraints

$$
\mathbf{V}^{T} \mathbf{V}=I_{n}
$$

almost surely.

- The probability distribution of $\mathbf{V}$ depends on a hyperparameter vector $\alpha=\left(\alpha_{1} \ldots \alpha_{m_{\alpha}}\right)^{T}$, where the dimension $m_{\alpha}$ is sufficiently small to enable the identification of $\alpha$ from the solution of a reduced-order inverse statistical problem. This problem is designed to minimize the discrepancies between the mean value and statistical fluctuations of the QoIs predicted using the SPROM constructed using V, and their counterparts based on the available data.

- The statistical fluctuations of $\mathbf{V}$ happen around its deterministic counterpart $V$.

The general procedure for constructing an SROB $\mathbf{V}$ that meets the above requirements was first presented in [5]. It is described in simplified form in Section III|C1 1 of this paper in order to keep it self-contained. The selection of the hyperparameters governing the probability distribution of this SROB is tailored in Section $[\mathrm{III} / \mathrm{C} 2$ to the context of the discrete, generalized EVP (II.1). The approach for identifying the hyperparameter vector $\alpha$ so that the SROB constructed in Section III]C1 can reproduce the mean value and statistical fluctuations of the QoIs computed from the available data is presented in Section III]D However, the SPROM to be built using the constructed SROB V and the Galerkin projection procedure is first presented in Section III B below.

\section{B. Stochastic projection-based reduced-order model}

The stochastic counterpart of the approximation (II.4) can be written as

$$
\mathbf{u} \approx \mathbf{V} \mathbf{u}_{r}
$$

where $\mathbf{u}$ and $\mathbf{u}_{r}$ are the stochastic counterparts of $u$ and $u_{r}$, respectively, and therefore represent the stochastic solution and its generalized coordinates in the basis $\mathbf{V}$, respectively. Substituting this approximation in problem III.1 and premultiplying both sides of the algebraic equation defining this problem by $\mathbf{V}^{T}$ leads to the following SPROM for the discrete, generalized EVP of interest

$$
\begin{aligned}
& \mathbf{K}_{r} \mathbf{u}_{r}=\varpi^{2} \mathbf{M}_{r} \mathbf{u}_{r} \\
& \text { where } \\
& \mathbf{K}_{r}=\mathbf{V}^{T} K \mathbf{V} \quad \text { and } \quad \mathbf{M}_{r}=\mathbf{V}^{T} M \mathbf{V}
\end{aligned}
$$

Because it is formulated using an SPROM of dimension $n \ll N$, the stochastic problem (III.2) can be rapidly solved to compute the realizations of the stochastic QoIs

$$
\left(\omega_{i}^{2}, \mathbf{u}_{i}\right) \approx\left(\varpi_{i}^{2}, \mathbf{V u} \mathbf{u}_{r_{i}}\right), \quad i=1, \cdots, m_{u} \leq n \ll N
$$

where $\left(\varpi_{i}^{2}, \mathbf{u}_{r_{i}}\right)$ is the $i$-th eigenpair solution of problem IIII.2.

\section{Stochastic reduced-order basis}

\section{Construction procedure}

From [III.1], it follows that the $N \times n$ SROB $\mathbf{V}$ must be constructed on a compact Stiefel manifold denoted here by $\mathbb{S}_{N, n}$. This can be done using the procedure summarized below and graphically depicted in Fig. 1. 
- Generate a centered random matrix $\mathbf{U} \in \mathbb{R}^{N \times n}$ (see the corresponding numerical procedure outlined below)

- Transform $\mathbf{U}$ into a random matrix $\mathbf{Z}$ that lies on the tangent space to $\mathbb{S}_{N, n}$ at $V, \mathcal{T}_{V} \mathbb{S}_{N, n}$

$$
\mathbf{Z}=\mathbf{U}-\frac{1}{2} V\left(V^{T} \mathbf{U}+\mathbf{U}^{T} V\right)
$$

Indeed, it follows from (III.3) that $\mathbf{Z}$ can also be written as

$$
\mathbf{Z}=\frac{1}{2} V\left(V^{T} \mathbf{U}-\mathbf{U}^{T} V\right)+\left(I_{N}-V V^{T}\right) \mathbf{U}
$$

where, from the orthonormality property of $V$, it follows that $V^{T}\left(I_{N}-V V^{T}\right) \mathbf{U}=V^{T} \mathbf{U}-V^{T} \mathbf{U}=0$. This shows that (III.4) expresses $\mathbf{Z}$ as the sum of one component in the subspace generated by $V$ and one component in the subspace that is $I$-orthogonal to $V$, and therefore $\mathbf{Z}$ lies on the tangent space to $\mathbb{S}_{N, n}$ at $V$.

- Transform $\mathbf{Z}$ into the matrix $\mathbf{V}$ which satisfies (III.1) and therefore lies on $\mathbb{S}_{N, n}$

$$
\mathbf{V}=(V+s \mathbf{Z})\left(I_{n}+s^{2} \mathbf{Z}^{T} \mathbf{Z}\right)^{-1 / 2}
$$

where $0 \leq s \leq 1$ can be treated as a hyperparameter of the probability model underlying $\mathbf{V}$. Indeed, from the orthonormality property of $V$ and the definition of $\mathbf{Z}$ given in (III.3), it follows that

$$
\begin{aligned}
\mathbf{V}^{T} \mathbf{V} & =\left(I_{n}+s^{2} \mathbf{Z}^{T} \mathbf{Z}\right)^{-1 / 2}\left(V^{T}+s \mathbf{Z}^{T}\right)(V+s \mathbf{Z})\left(I_{n}+s^{2} \mathbf{Z}^{T} \mathbf{Z}\right)^{-1 / 2} \\
& =\left(I_{n}+s^{2} \mathbf{Z}^{T} \mathbf{Z}\right)^{-1 / 2}(I_{n}+s \underbrace{\left(V^{T} \mathbf{Z}+\mathbf{Z}^{T} V\right)}_{0}+s^{2} \mathbf{Z}^{T} \mathbf{Z})\left(I_{n}+s^{2} \mathbf{Z}^{T} \mathbf{Z}\right)^{-1 / 2} \\
& =I_{n}
\end{aligned}
$$

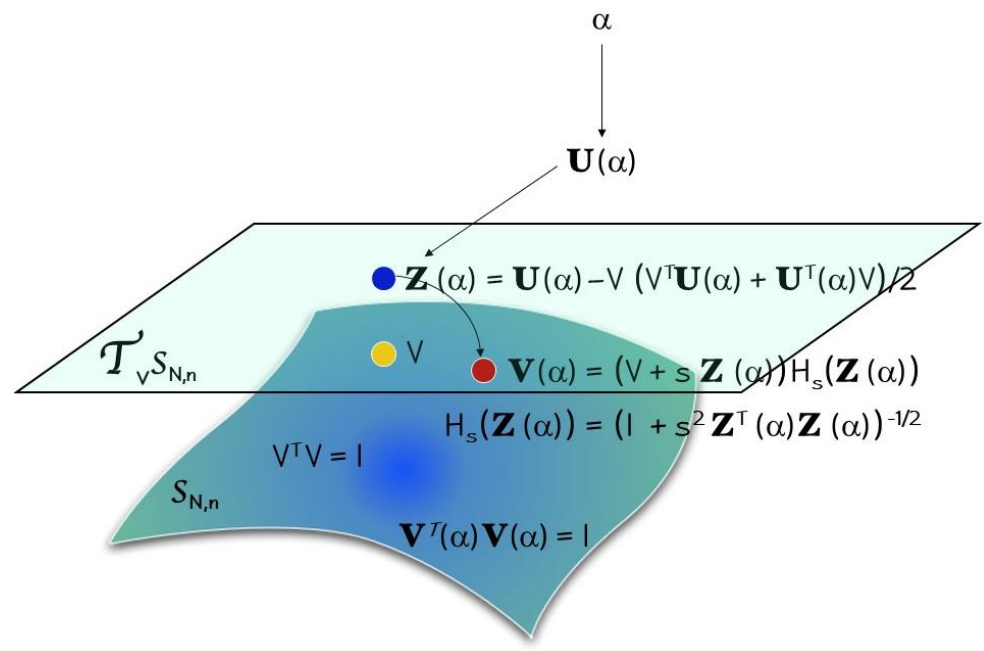

Fig. 1 Construction of the SROB $\mathrm{V}$ on the compact Stiefel manifold $\mathbb{S}_{N, n}$ (construction procedure shown for $\left.V / V^{T} V=I\right)$.

Note that the definition (III.5) given above shows that:

- The statistical fluctuations of $\mathbf{V}$ happen around its deterministic counterpart $V$, as desired. In particular, for $s=0$, $\mathbf{V}=V$. However, it is noted that in general, $E(\mathbf{V}) \neq V$ (see [5]).

- The real number $s$ controls the level of fluctuations of $\mathbf{V}$ in the tangent plane to $\mathbb{S}_{N, n}$, around $V \in \mathbb{S}_{N, n}$.

Note also that whereas the matrices $\mathbf{U}$, and $\mathbf{Z}$ leading to the construction of $\mathbf{V}$ are random matrices, the construction process of $\mathbf{V}$ itself is deterministic. In particular, no matter how large is the excursion of $V+s \mathbf{Z}$ in the tangent plane to $\mathbb{S}_{N, n}$ around $V \in \mathbb{S}_{N, n}$, the resulting matrix $\mathbf{V}=(V+s \mathbf{Z})\left(I_{n}+s^{2} \mathbf{Z}^{T} \mathbf{Z}\right)^{-1 / 2}$ lies on the manifold $\mathbb{S}_{N, n}$. Hence, for all these reasons, the SROB V defined in (III.5) satisfies all requirements formulated in Section III]A. 


\section{Random matrix $\mathbf{U}$ and associated hyperparameters}

From (III.3) and (III.5), it follows that if $\mathbf{U}=0$, then $\mathbf{Z}=0$ and $\mathbf{V}=V$. This suggests that, consistently with the idea that the statistical fluctuations of $\mathbf{V}$ should occur around its deterministic counterpart $V$, $\mathbf{U}$ should be a centered random matrix $(E[\mathbf{U}]=0$, where $E$ designates the mathematical expectation of a random variable).

The covariance tensor $\mathbb{C}_{j k j^{\prime} k^{\prime}}=E\left[\mathbf{U}_{j k} \mathbf{U}_{j^{\prime} k^{\prime}}\right]$ is a fourth-order symmetric tensor with $N n(N n+1) / 2=O\left(N^{2} n^{2}\right)$ coefficients that can be considered as parameterization coefficients. As already mentioned in Section III]A, part of the idea here is to use such coefficients as hyperparameters and determine them so that the mean value and statistical fluctuations of the eigenvalue solutions of the SPROM (III.2) match target values obtained from available data. However, working for this purpose with $O\left(N^{2} n^{2}\right)$ hyperparameters is not computationally feasible. Hence, in order to reduce its number of hyperparameters, the random matrix $\mathbf{U}$ is constructed so that

$$
\mathbb{C}_{j k j^{\prime} k^{\prime}}=E\left[\mathbf{U}_{j k} \mathbf{U}_{j^{\prime} k^{\prime}}\right]=C_{N}(\beta)_{j j^{\prime}} c_{n_{k k^{\prime}}}
$$

where $\beta$ is a hyperparameter, $C_{N}(\beta) \in \mathbb{R}^{N \times N}$ controls the correlations between the components of each column vector of $\mathbf{U}$, and the matrix $c_{n} \in \mathbb{R}^{n \times n}$, which is a Symmetric Positive Definite (SPD) matrix, controls the correlations between the column vectors of $\mathbf{U}$. For a given application, the construction of $C_{N}(\beta)$ is given by a mathematical process whose description can be found in Appendix D of Reference [5]. According to the Cholesky decomposition, $c_{n}$ can be written as

$$
c_{n}=\tau^{T} \tau
$$

where $\tau \in \mathbb{R}^{n \times n}$ is an upper triangular matrix. This approach for constructing $\mathbf{U}$ reduces the number of hyperparameters from $N n(N n+1) / 2$ to $1+n(n+1) / 2=O\left(n^{2}\right)$.

In Reference [5], it is shown that both objectives $E[\mathbf{U}]=0$ and (III.6) can be achieved by constructing a random matrix $\mathbf{G} \in \mathbb{R}^{N \times n}$ that: is centered $(E[\mathbf{G}]=0)$; is computed by semi-discretizing a random field defined at the continuous level of the governing equation of the problem of interest; verifies $E\left[\mathbf{G}_{j k} \mathbf{G}_{j^{\prime} k^{\prime}}\right]=C_{N}(\beta)_{j j^{\prime}} \delta_{k k^{\prime}}$; and generates the random matrix $\mathbf{U}$ as follows

$$
\mathbf{U}=\mathbf{G}(\beta) \tau
$$

Given that $s$ in III.5) is also a hyperparameter, it follows that the probability model underlying $\mathbf{V}$ defined so far has $2+n(n+1) / 2$ hyperparameters: $s, \beta$, and the coefficients of the upper triangular matrix $\tau$. In the remainder of this paper, these parameters of prior distribution are referred to as the main hyperparameters.

\section{Additional hyperparameter for reducing the bias of the mean value}

For computing the lowest eigenvalues, it is sometimes difficult to identify optimal values of the main hyperparameters described in Section III|C|2 - that is, values of these parameters that sufficiently reduce the bias between the mean value $E\left\{\varpi_{j}^{2}\right\}$ estimated using the SPROM (III.2 and the corresponding value extracted from the available data. To address this issue, an additional, strictly positive hyperparameter $s_{M}>0$ belonging to a real interval in the neighborhood of 1 is introduced here as follows. Specifically, each stochastic QoI $\varpi_{j}^{2}$ in the SPROM IIII.2) is re-written as

$$
\varpi_{j}^{2}=s_{M} \widehat{\varpi}_{j}^{2}, \quad j=1, \ldots, m_{u}
$$

and the statistics of the random eigenvalues are constructed for $\widehat{\varpi}_{j}^{2}$ instead of $\varpi_{j}^{2}$. Noting that $E\left\{\varpi_{j}^{2}\right\}=s_{M} E\left\{\widehat{\varpi}_{j}^{2}\right\}$, it follows that the purpose of the hyperparameter $s_{M}$ is to enable a better global fit of the mean values $\left\{E\left\{\varpi_{j}^{2}\right\}, j=\right.$ $\left.1, \cdots, m_{u}\right\}$.

In summary, for the class of generalized eigenvalue problems considered in this paper, the hyperparameters governing the probability distribution of the SROB $\mathbf{V}$ are:

- The hyperparameter $s$ which is a real number and controls the level of fluctuations of $\mathbf{V}$ in the tangent plane to $\mathbb{S}_{N, n}$, around $V \in \mathbb{S}_{N, n}($ see $($ III.5) $)$.

- The hyperparameter $\beta$ controlling the matrix $C_{N}(\beta)_{j j^{\prime}}$ (see (III.6)).

- The $n(n+1) / 2$ hyperparameters of the matrix $\tau$ (see (III.8) represented here by the vector vec $(\tau)$.

- The hyperparameter $s_{M}$ introduced above (see (III.9) ).

These parameters of prior distribution are collected here in the hyperparameter vector

$$
\alpha=\left(s, s_{M}, \beta, \operatorname{vec}(\tau)\right)
$$


In principle, the upper triangular matrix $\tau$ (III.7) can be sparsified. Thus, an upper bound for the total number of hyperparameters described above is $m_{\alpha}^{u b}=3+n(n+1) / 2$, and a lower bound is $m_{\alpha}^{l b}=4$. Consequently, the dimension of the hyperparameter vector [III.10) satisfies in general

$$
4 \leq m_{\alpha} \leq 3+n(n+1) / 2
$$

\section{Identification of the hyperparameters}

As already stated in Section III|A, the identification of the hyperparameter vector $\alpha$ is to be achieved so that the mean value and statistical fluctuations of the QoIs predicted using the SPROM match target values obtained using the available data. This can be performed using the maximum likelihood method, or a nonlinear Least-Squares (LS) method for the QoIs. For example, a nonlinear LS method can be formulated as described below, for both types of model-form uncertainties outlined in Section!

Let

$$
o(\varrho)=\left(o_{1}(\varrho), \ldots, o_{j}(\varrho), \ldots, o_{m_{o}}(\varrho)\right)
$$

denote the vector QoI (or system observation). For example, for problem (III.1), this vector can be $o(\varrho)=$ $\left(\omega_{1}^{2}(\varrho), \ldots, \omega_{j}^{2}(\varrho), \ldots, \omega_{m_{u}}^{2}(\varrho)\right)\left(m_{o}=m_{u} \leq n \ll N\right)$. For problem III.5), it can be $o(\varrho)=\left(\varpi_{1}^{2}(\varrho), \ldots, \varpi_{j}^{2}(\varrho)\right.$, $\left.\ldots, \varpi_{m_{u}}^{2}(\varrho)\right)\left(m_{o}=m_{u} \leq n \ll N\right)$, and for problem $\left[\right.$ III.2, it can be $\boldsymbol{o}(\varrho)=\left(\varpi_{1}^{2}(\varrho), \ldots, \varpi_{j}^{2}(\varrho), \ldots, \varpi_{m_{u}}^{2}(\varrho)\right)$ $\left(m_{o}=m_{u} \leq n \ll N\right)$.

Let also $J(\alpha)$ be the cost function defined as

$$
J(\alpha)=w_{J} J_{\text {mean }}(\alpha)+\left(1-w_{J}\right) J_{\text {std }}(\alpha)
$$

where $w_{J}$ is a weight satisfying $0 \leq w_{J} \leq 1$, and $J_{\text {mean }}(\alpha)$ and $J_{\text {std }}(\alpha)$ are two functions constructed for controlling the identification of $\alpha$ with respect to the mean value and statistical fluctuations, respectively. Specifically, $J_{\text {mean }}(\alpha)$ and $J_{\text {std }}(\alpha)$ are defined as

$$
\begin{aligned}
J_{\text {mean }}(\alpha) & =\frac{1}{c_{\text {mean }}\left(\varrho_{1}, \ldots, \varrho_{n_{\varrho}}\right)} \sum_{k=1}^{n_{\varrho}}\left\|o^{\mathrm{ref}}\left(\varrho_{k}\right)-E\left[\boldsymbol{o}\left(\varrho_{k}, \alpha\right)\right]\right\|^{2} \\
\text { and } & \\
J_{\text {std }}(\alpha) & =\frac{1}{c_{\text {std }}\left(\varrho_{1}, \ldots, \varrho_{n_{\varrho}}\right)} \sum_{k=1}^{n_{\varrho}}\left\|v^{\mathrm{ref}}\left(\varrho_{k}\right)-\mathbf{v}\left(\varrho_{k}, \alpha\right)\right\|^{2}
\end{aligned}
$$

where:

- $n_{\varrho}$ denotes as before the number of sampled parameter points $\varrho_{k} \in \Omega_{\varrho}$.

- $E$ denotes as before the mathematical expectation.

- The definition of $o^{\text {ref }}$ depends on the type of available data, which dictates the types of model-form uncertainties that can be accounted for by the proposed approach for modeling model-form uncertainties:

- If only high-dimensional data - that is, data generated using an HDM - is available, $o^{\text {ref }}$ corresponds to the QoIs of the parametric problem (II.1) formulated using an HDM. In this case, only the errors/uncertainties due to model reduction are taken into account by the proposed approach for modeling model-form uncertainties.

- If experimental data are available:

* If these data are nonstatistical experimental data, then

$$
o^{\text {ref }}=o^{\text {exp }}
$$

where $o^{\text {exp }}$ is extracted from these available data.

* If these data are statistical experimental data, then

$$
o^{\mathrm{ref}}=E\left[\boldsymbol{o}^{\mathrm{exp}}\right]
$$

where $\boldsymbol{o}^{\text {exp }}$ is extracted from these available data.

In either case of experimental data described above, both of the errors/uncertainties due to model reduction and the uncertainties inherited from the parametric HDM are taken into account by the proposed approach for modeling model-form uncertainties. 
- $v^{\text {ref }}\left(\varrho_{k}\right)=\left(v_{1}^{\text {ref }}\left(\varrho_{k}\right), \ldots, v_{j}^{\text {ref }}\left(\varrho_{k}\right), \ldots, v_{m_{o}}^{\text {ref }}\left(\varrho_{k}\right)\right), k=1, \cdots, n_{\varrho}$, is defined as follows:

- If only high-dimensional data is available, then

$$
v_{j}^{\text {ref }}\left(\varrho_{k}\right)=\gamma\left|o_{j}^{\text {ref }}\left(\varrho_{k}\right)-o_{j}\left(\varrho_{k}\right)\right|, \quad j=1, \ldots m_{o}
$$

where $\gamma>0$ is a tunable control parameter, $o_{j}^{\text {ref }}\left(\varrho_{k}\right)=\omega_{j}^{2}\left(\varrho_{k}\right)$ and $o_{j}\left(\varrho_{k}\right)=\varpi_{j}^{2}\left(\varrho_{k}\right)$.

- If nonstatistical experimental data are available, then

$$
v_{j}^{\mathrm{ref}}\left(\varrho_{k}\right)=\gamma\left|o_{j}^{\mathrm{ref}}\left(\varrho_{k}\right)-o_{j}\left(\varrho_{k}\right)\right|, \quad j=1, \ldots m_{o}
$$

where, again, $\gamma>0$ is a tunable control parameter and $o_{j}\left(\varrho_{k}\right)=\varpi_{j}^{2}\left(\varrho_{k}\right)$, but $o_{j}^{\text {ref }}\left(\varrho_{k}\right)=o_{j}^{\exp }\left(\varrho_{k}\right)$ (from (III.13).

- If statistical experimental data are available, then

$$
v_{j}^{\text {ref }}\left(\varrho_{k}\right)=\sqrt{E\left(\boldsymbol{o}_{j}^{\exp }\left(\varrho_{k}\right)^{2}\right)-\left[E\left(\boldsymbol{o}_{j}^{\exp }\left(\varrho_{k}\right)\right)\right]^{2}}, \quad j=1, \ldots m_{o}
$$

- $\mathbf{v}\left(\varrho_{k}, \alpha\right)=\left(\mathbf{v}_{1}\left(\varrho_{k}, \alpha\right), \ldots, \mathbf{v}_{m_{o}}\left(\varrho_{k}, \alpha\right)\right)$, where

$$
\mathbf{v}_{j}\left(\varrho_{k}, \alpha\right)=\sqrt{E\left(\boldsymbol{o}_{j}^{2}\left(\varrho_{k}, \alpha\right)\right)-\left[E\left(\boldsymbol{o}_{j}\left(\varrho_{k}, \alpha\right)\right)\right]^{2}}, \quad j=1, \ldots m_{o}
$$

- The positive constants $c_{\text {mean }}\left(\varrho_{1}, \ldots, \varrho_{n_{\varrho}}\right)$ and $c_{\text {std }}\left(\varrho_{1}, \ldots, \varrho_{n_{\varrho}}\right)$ are defined as

$$
c_{\text {mean }}\left(\varrho_{1}, \ldots, \varrho_{n_{\varrho}}\right)=\sum_{k=1}^{n_{\varrho}}\left\|o^{\text {ref }}\left(\varrho_{k}\right)\right\|^{2}, \quad c_{\text {std }}\left(\varrho_{1}, \ldots, \varrho_{\varrho}\right)=\sum_{k=1}^{n_{\varrho}}\left\|v_{j}^{\text {ref }}\left(\varrho_{k}\right)\right\|^{2}
$$

It follows that in the definitions (III.11) and III.12) of the cost function $J(\alpha), o^{\text {ref }}$ is the target for the mean value with a weight $w_{J}$, and $v^{\text {ref }}$ is the target for the standard deviation with a weight $1-w_{J}$.

The identification of the hyperparameter vector consists in calculating $\alpha$ such that

$$
\alpha=\arg \min _{\vartheta} J(\vartheta)
$$

\section{Hyperreduction}

The reader is reminded that the notation $\bullet(\varrho)$ emphasizes the dependence of the quantity $\bullet$ on the parameter vector $\varrho \in \Omega_{\varrho}$, and that occasionally, this dependence is not explicitly highlighted in the remainder of this paper for the sake of brevity.

Whichever optimization strategy is chosen for solving the minimization problem (III.14) to determine the hyperparameter vector $\alpha$, it is bound to be an iterative one. From [III.2], it follows that at each $k$-th iteration of such a strategy, the stochastic reduced stiffness and mass matrices

$$
\mathbf{K}_{r}(\vartheta)=\mathbf{V}^{T}(\vartheta) K \mathbf{V}(\vartheta) \quad \text { and } \quad \mathbf{M}_{r}(\vartheta)=\mathbf{V}^{T}(\vartheta) K \mathbf{V}(\vartheta)
$$

must be recomputed so that: multiple independent realizations of the QoIs may be regenerated by solving different instances of the SPROM (III.2) using the updated value of the hyperparameter vector $\vartheta$; and $J_{\text {mean }}$ and $J_{\text {std }}$ (III.12) may be reevaluated accordingly.

Each reconstruction of $\mathbf{K}_{r}(\vartheta)\left(\mathbf{M}_{r}(\vartheta)\right)$ requires:

- (R1) First, reconstructing the SROB V $(\vartheta) \in \mathbb{R}^{N \times n}$ on the compact Stiefel manifold $\mathbb{S}_{N, n}$, as explained in Section IIICC1 and summarized in Fig. 11.

- (R2) Then, reconstructing the new reduced matrix $\mathbf{K}_{r}(\vartheta)\left(\mathbf{M}_{r}(\vartheta)\right)$, which incurs the computation of the matrix-matrix-matrix product $\mathbf{V}^{T}(\vartheta) K \mathbf{V}(\vartheta)\left(\mathbf{V}^{T}(\vartheta) M \mathbf{V}(\vartheta)\right)$, where $K(M) \in \mathbb{R}^{N \times N}$, respectively.

Unfortunately, the computational complexity of each of the above two requirements, and therefore that of rebuilding each of $\mathbf{K}_{r}(\vartheta)$ and $\mathbf{M}_{r}(\vartheta)$, does not scale only with the size of the SPROM $n$, but also with that of the HDM $N$. Therefore, reconstructing each of these two reduced matrices at the beginning of each iteration of the solution of the minimization problem (III.14) constitutes a serious computational bottleneck. 
In model reduction, such a bottleneck, which is typically encountered in deterministic nonlinear PROM computations but occurs here in stochastic linear PROM ones, is avoided by hyperreducing the PROM - that is, by approximating quantities such as $\mathbf{K}_{r}$ and $\mathbf{M}_{r}$ using a method whose computational complexity scales only with the dimension $n$ of the SPROM. Here, the ECSW (Energy Conserving Sampling and Weighting) [8] method is chosen for this purpose because for structural dynamics problems: its superior performance relative to alternative hyperreduction methods has been amply demonstrated (for example, see [11]); and this hyperreduction method preserves the Lagrangian structure associated with Hamilton's principle for second-order dynamical systems (see also [11]).

In the deterministic case - for example, for the PROM (II.5) - the reduced stiffness and mass matrices defined in (II.6) can be written as

$$
K_{r}=V^{T} K V=\sum_{e \in \mathcal{E}}\left(L^{e} V\right)^{T} K^{e}\left(L^{e} V\right) \quad \text { and } \quad M_{r}=V^{T} M V=\sum_{e \in \mathcal{E}}\left(L^{e} V\right)^{T} M^{e}\left(L^{e} V\right)
$$

where $L^{e}$ is the standard localization operator to the element $e$ of the FE mesh underlying the given FE HDM, and $\mathcal{E}=\left\{e_{1}, \cdots, e_{n^{e}}\right\}$ is the set of all $n^{e}$ elements of this mesh. The ECSW hyperreduction method approximates the above matrices using a quadrature rule that can be written as

$$
K_{r} \approx \widetilde{K}_{r}=\sum_{e \in \widetilde{\mathcal{E}}} \xi^{e}\left(L^{e} V\right)^{T} K^{e}\left(L^{e} V\right) \quad \text { and } \quad M_{r} \approx \widetilde{M}_{r}=\sum_{e \in \widetilde{\mathcal{E}}} \xi^{e}\left(L^{e} V\right)^{T} M^{e}\left(L^{e} V\right)
$$

where $\widetilde{\mathcal{E}} \subset \mathcal{E}$ is a subset of the elements of the FE mesh that is sampled by ECSW, and for each element $e \in \widetilde{\mathcal{E}}$, $\xi^{e} \in \mathbb{R}_{>0}$ is a positive scalar weight that is also determined by ECSW. In other words, the ECSW approximation (IV.2) is a quadrature approximation where each element $e \in \widetilde{\mathcal{E}}$ plays the role of a quadrature "point", and the corresponding coefficient $\xi^{e} \in \mathbb{R}_{>0}$ plays the role of a quadrature "weight". The positivity requirement for $\xi^{e}$ is enforced so that $\xi^{e}\left(L^{e} V\right)^{T} K^{e}\left(L^{e} V\right)$ preserves the meaning of a stiffness matrix and therefore remains a symmetric positive semi-definite matrix. To this end, the ECSW method samples the set of elements $\mathcal{E}$ to construct the reduced mesh with $\tilde{n}^{e}$ elements defining $\widetilde{\mathcal{E}} \subset \mathcal{E}$, and determines the associated weight vector $\xi=\left(\xi_{e_{1}}, \ldots, \xi_{\tilde{n}_{e}}\right)$ by minimizing an objective function that promotes:

- Sparsity $\left(\tilde{n}^{e} \ll n^{e}\right)$ in order to reduce the computational complexity of the approximations (IV.2].

- Accuracy for a given set of training data that is relevant to the quantities being hyperreduced. Substituting the approximations (IV.2) into the PROM (II.5) yields the HPROM for the discrete, generalized EVP (II.1)

$$
\widetilde{K}_{r}(\varrho) u_{r}(\varrho)=\varpi^{2}(\varrho) \widetilde{M}_{r}(\varrho) u_{r}(\varrho)
$$

In [8] and [11], ECSW was developed for the hyperreduction of deterministic PROMs associated with various nonlinear versions of the equation of dynamic equilibrium (II.2) such as, for example, the governing equation

$$
M(\varrho) \ddot{u}(\varrho, t)+f^{i n t}(u(\varrho, t), \dot{u}(\varrho, t), \Xi(t))=f^{e x t}(\varrho, t)
$$

where $f^{\text {int }}$ denotes the internal force vector, and $\Xi(t) \in \mathbb{R}^{n_{\text {int }}}$ is a time-dependent vector of $n_{\text {int }}$ internal variables associated with some nonlinear constitutive relation. Using the notation of this paper, the nonlinear PROM associated with the above equation can be written as

$$
M_{r} \ddot{u}_{r}+V^{T} f^{i n t}\left(V u_{r}(\varrho, t), V \dot{u}_{r}(\varrho, t), \Xi(t)\right)=V^{T} f^{e x t}(\varrho, t)
$$

(Specifically, ECSW was developed in [8] for hyperreducing the reduced internal force vector $f_{r}^{\text {int }}=V^{T} f^{\text {int }}$.) The time-discretization of this nonlinear PROM by an implicit scheme leads to a discrete system of nonlinear equations whose solution by a Newton method gives rise at each iteration to a linearized system of equations of the form

$$
\begin{array}{rcc}
K_{r}^{d y n} \Delta u_{r} & =\quad g_{r} \\
& \text { where } & \\
K_{r}^{d y n}=V^{T} K^{d y n} V \quad \text { and } & g_{r}=V^{T} g
\end{array}
$$

Here, the symbol $\Delta$ designates the increment of a quantity, and the exact expressions of the dynamic tangent stiffness matrix $K^{d y n} \in \mathbb{R}^{N \times N}$ and the right-hand side vector $g \in \mathbb{R}^{N}$ depend on the linearization of the internal force vector and the time-discretization scheme. Specifically, the approximation of $K_{r}$ given in [IV.2) was developed in [8] for 
the matrix $K_{r}^{d y n}$ of IV.3 which has the same algebraic form and structure. Furthermore, training for finding the reduced mesh $\widetilde{\mathcal{E}}$ and the associated weight vector $\xi$ was performed in [8] using a precomputed set of internal forces $f^{\text {int }}\left(V\left(V^{T} V\right)^{-1} V^{T} u^{s}\right)$, where $u^{s}$ is the $s$-th collected solution snapshot of III.2 for some sampled value of $\varrho \in \Omega_{\varrho}$. For an orthonormal basis $V\left(V^{T} V=I_{n}\right)$, this training data can be simply written as $f^{\text {int }}\left(V V^{T} u^{s}\right)$. Hence, the training procedure developed in [8] consisted in finding the smallest subset $\widetilde{\mathcal{E}}$ and the associated weight vector $\xi$ for which the approximation of each reduced force $f_{r}^{i n t}\left(V V^{T} u^{s}\right)=V^{T} f^{i n t}\left(V V^{T} u^{s}\right)$ using the same quadrature rule as that implied by (IV.2) - that is,

$$
f_{r}^{i n t}=V^{T} f^{i n t}\left(V V^{T} u^{s}\right) \approx \tilde{f}_{r}^{i n t}\left(V V^{T} u^{s}\right)=\sum_{e \in \widetilde{\mathcal{E}}} \xi^{e}\left(L^{e} V\right)^{T} f^{i n t}\left(\left(L^{e} V\right)\left(L^{e} V\right)^{T} u^{s}\right), \quad s=1, \cdots, n_{s}
$$

is sufficiently accurate in the sense of

$$
\left\|f_{r}^{\text {int }}\left(V V^{T} u^{s}\right)-\tilde{f}_{r}^{i n t}\left(V V^{T} u^{s}\right)\right\|_{2} \leq \epsilon\left\|f_{r}^{i n t}\left(V V^{T} u^{s}\right)\right\|_{2}, \quad s=1, \cdots, n_{s}
$$

where $\epsilon$ is some small tolerance. Hence, this training procedure can be formulated as the solution of the following minimization problem

$$
\xi=\arg \min _{\zeta \in \mathbb{R}_{\geq 0}^{n_{e}}}\|\zeta\|_{0} \quad \text { such that } \quad\|G \zeta-b\|_{2} \leq \varepsilon\|b\|_{2}
$$

where the notation $\zeta \in \mathbb{R}_{>0}^{n_{e}}$ implies that, in the above formulation, the weight vector $\xi$ is defined for the entire mesh associated with the FE HDM and represented by the set of elements $\mathcal{E},\|\zeta\|_{0}$ counts the number of nonzero entries in the vector $\zeta, G$ and $b$ arise from the matrix form of (IV.4), and $\varepsilon$ is a specified small tolerance. Using this formulation, it follows that the reduced mesh constructed by the ECSW hyperreduction method is the subset of elements $\widetilde{\mathcal{E}} \subset \mathcal{E}$ for which the entries of the vector $\xi \in \mathbb{R}_{\geq 0}^{n_{e}}$ computed by solving the minimization problem (IV.5) are nonzero.

Unfortunately, the minimization problem (IV.5) is NP-hard. For this reason, it was argued in [8] and shown in [12] that the alternative, non-negative least squares minimization problem

$$
\xi \approx \arg \min _{\zeta \in \mathbb{R}_{\geq 0}^{n_{e}}} \frac{1}{2}\|G \zeta-b\|_{2}^{2}
$$

delivers an excellent if not the best approximate solution of problem (IV.5).

In the nondeterministic case, hyperreduction must be applied to both requirements (R1) and (R2) to be computationally effective. To this end, it is first noted that from (IV.1) and (IV.2), it follows that applying ECSW to the requirement (R2) implies approximating the reduced stochastic matrices $\mathbf{K}_{r}(\vartheta)$ and $\mathbf{M}_{r}(\vartheta)$ at each iteration of the solution of the minimization problem (III.14) as follows

$$
\begin{array}{cc}
\mathbf{K}_{r}(\vartheta) \approx \widetilde{\mathbf{K}}_{r}(\vartheta, \xi) & =\sum_{e \in \widetilde{\mathcal{E}}} \xi^{e}\left(L^{e} \mathbf{V}(\vartheta)\right)^{T} \mathbf{K}^{e}\left(L^{e} \mathbf{V}(\vartheta)\right) \\
\mathbf{M}_{r}(\vartheta) \approx \widetilde{\mathbf{M}}_{r}(\vartheta, \xi) & \text { and } \sum_{e \in \widetilde{\mathcal{E}}} \xi^{e}\left(L^{e} \mathbf{V}(\vartheta)\right)^{T} \mathbf{M}^{e}\left(L^{e} \mathbf{V}(\vartheta)\right)
\end{array}
$$

where the dependence of $\widetilde{\mathbf{K}}_{r}$ and $\widetilde{\mathbf{M}}_{r}$ on $\xi$ is emphasized. These approximations transform the SPROM III.2 into the following SHPROM for the discrete, generalized EVP (II.1)

$$
\widetilde{\mathbf{K}}_{r} \mathbf{u}_{r}=\varpi^{2} \widetilde{\mathbf{M}}_{r} \mathbf{u}_{r}
$$

In this case, the training procedure outlined above for determining the weight vector $\xi$ can be extended to the construction of the above SHPROM by noting that the stochastic counterpart of the inequality (IV.4) can be written in the context of the time domain equivalent of problem (III.2) as

$$
\begin{aligned}
\left\|\mathbf{K}_{r}(\vartheta) \mathbf{V}(\vartheta) \mathbf{V}^{T}(\vartheta) \mathbf{u}^{s}-\widetilde{\mathbf{K}}_{r}(\vartheta, \xi) \mathbf{V}(\vartheta) \mathbf{V}^{T}(\vartheta) \mathbf{u}^{s}\right\|_{2} \leq \varepsilon\left\|\mathbf{K}_{r}(\vartheta) \mathbf{V}(\vartheta) \mathbf{V}^{T}(\vartheta) \mathbf{u}^{s}\right\|_{2}, \quad s=1, \cdots, n_{s} \\
\left\|\mathbf{M}_{r}(\vartheta) \mathbf{V}(\vartheta) \mathbf{V}^{T}(\vartheta) \ddot{\mathbf{u}}^{s}-\widetilde{\mathbf{M}}_{r}(\vartheta, \xi) \mathbf{V}(\vartheta) \mathbf{V}^{T}(\vartheta) \ddot{\mathbf{u}}^{s}\right\|_{2} \leq \varepsilon\left\|\mathbf{M}_{r}(\vartheta) \mathbf{V}(\vartheta) \mathbf{V}^{T}(\vartheta) \ddot{\mathbf{u}}^{s}\right\|_{2}, \quad s=1, \cdots, n_{s}
\end{aligned}
$$


where the first and second of the above two inequalities state that the hyperreduction of the internal and inertia force vectors using the ECSW method are sufficiently accurate, respectively. Hence, following the same reasoning as in the deterministic case, the training procedure for determining the weight vector $\xi$ underlying the construction of the SHPROM (IV.8) using the ECSW hyperreduction method can be formulated as the solution of the following minimization problem

$$
\xi \approx \arg \min _{\zeta \in \mathbb{R}_{\geq 0}^{n_{e}}} \frac{1}{2}\|\mathbf{G}(\vartheta) \zeta-\mathbf{b}(\vartheta)\|_{2}^{2}
$$

where $\mathbf{G}$ and $\mathbf{b}$ arise from the matrix form of the stacked inequalities (IV.9).

Next, it is also noted that from (IV.7), it follows that applying ECSW to the requirement (R1) essentially implies constructing only the trace of the $\operatorname{SROB} \mathbf{V}(\vartheta)$ on the reduced mesh defined by $\widetilde{\mathcal{E}}$. Since $\widetilde{\mathcal{E}}$ is identified by the nonzero entries of $\xi \in \mathbb{R}_{\geq 0}^{n_{e}}$, if follows that $\widetilde{\mathcal{E}}=\widetilde{\mathcal{E}}(\xi)$. Hence, the trace of $\mathbf{V}(\vartheta)$ on $\widetilde{\mathcal{E}}$, which is denoted here by $\mathbf{V}_{\widetilde{\mathcal{E}}}$, can be written as

$$
\mathbf{V}_{\widetilde{\mathcal{E}}}=\mathbf{V}_{\widetilde{\mathcal{E}}}(\vartheta, \xi)
$$

It follows that the counterpart of the minimization problem (III.14) based on the SHPROM (IV.8) is given by

$$
\alpha=\arg \min _{\vartheta} J(\vartheta, \xi)
$$

In summary, applying the ECSW hyperreduction method to both requirements (R1) and (R2) - or equivalently, to the construction of the SHPROM (IV.8) - leads in principle to the following $\xi-\alpha$ coupled minimization problem

$$
\begin{aligned}
& \xi \approx \arg \min _{\zeta \in \mathbb{R}_{\geq 0}^{n e}} \frac{1}{2}\|\mathbf{G}(\alpha) \zeta-\mathbf{b}(\alpha)\|_{2}^{2} \\
& \alpha=\arg \min _{\vartheta} J(\vartheta, \xi)
\end{aligned}
$$

However, for the sake of computational efficiency, the above coupled minimization problem (IV.10) is decoupled here and therefore simplified as follows

$$
\begin{aligned}
\xi & \approx \arg \min _{\zeta \in \mathbb{R}_{\geq 0}^{n_{e}}} \frac{1}{2}\|G \zeta-b\|_{2}^{2} \\
\alpha & =\arg \min _{\vartheta} J(\vartheta, \xi)
\end{aligned}
$$

In other words, hyperreduction via the ECSW method is first applied to both requirements (R1) and (R2) using the same reduced mesh $\widetilde{\mathcal{E}}$ obtained for the hyperreduction of the deterministic PROM III.5) and the associated weight vector $\xi$. Then, the identification of the hyperparameter vector $\alpha$ is performed using this reduced mesh, the associated weight vector, and the approximate reduced stiffness and mass matrices based on them (IV.7) to construct the SHPROM (IV.8).

\section{Application}

Here, the potential of the nonparametric probabilistic method described in this paper for performing model-form UQ is illustrated with what-if? vibration analysis scenarios associated with shape changes of a jet engine nozzle. The proposed approach is applied to model and quantify the model-form uncertainties associated with the FE HDMs generated for modeling the structural vibrations of this nozzle. To this end, two shape-parametric FE HDMs are built: one with a higher fidelity to serve as a surrogate experimental system, and another one with a lesser fidelity to simulate model-form uncertainties. A shape-parametric SHPROM is constructed for the latter HDM to accelerate the stochastic computations incurred by the proposed nonparametric probabilistic method for model-form UQ. All additional uncertainties introduced by this auxiliary SHPROM, including those associated with the finite sampling of the shape-parameter domain $\Omega_{\varrho}$ and the errors introduced by basis truncation and hyperreduction are accounted for. UQ analysis is performed for the eigenvalues of the modeled structural systems associated with two shape-parameter points in $\Omega_{\varrho}$.

All FE structural computations reported here, whether at the HDM, PROM, or HPROM level, are performed using the FE structural analyzer AERO-S [13, 14]. On the other hand, all constructions of SHPROMs and all computations performed using SHPROMs are carried out using MATLAB $^{\odot}$. 


\section{A. Parametric jet engine nozzle and FE representations}

The engine nozzle considered here has an axisymmetric cylindrical shape. It consists of five circular sections delimited by six baffles on the exterior of the nozzle, and four exterior stringers that run from the inlet to the outlet (see Fig. 2). Furthermore, this shape is parameterized by a scaling factor $l$ along the axis of axisymmetry (the $x$-axis in Fig. 27, and a scaling factor $r$ in the directions perpendicular to this axis. These two scaling factors define a shape-parameter domain $\Omega_{\varrho}=(l, r)$.

The nominal main length of the nozzle is $L=0.536 \mathrm{~m}$. Its nominal radius at its widest circular cross section is $R=0.42 \mathrm{~m}$. Its thickness, which varies along the axis of axisymmetry but is constant in a cross section, decreases from $6.72 \times 10^{-4} \mathrm{~m}$ at the outlet to $5.00 \times 10^{-4} \mathrm{~m}$ in the middle section; then, it increases from its value at the middle section to the value of $1.32 \times 10^{-3} \mathrm{~m}$ at the outlet. The inlet cross section of the nozzle is clamped.

For each considered shape configuration of this nozzle - that is, for each considered value of the shape-parameter vector $\rho=(l, r)^{T}$ - two FE structural dynamics HDMs are constructed with 4,640 nodes and 9,200 triangular anisotropic AQR shell elements with 6 degrees of freedom (dofs) per node. Each of these HDMs has 27,240 unconstrained dofs and therefore $N=27,240$ - and 600 constrained dofs where the boundary conditions are applied. They differ only in how they represent the true thickness of the nozzle, which is discussed in the next section.

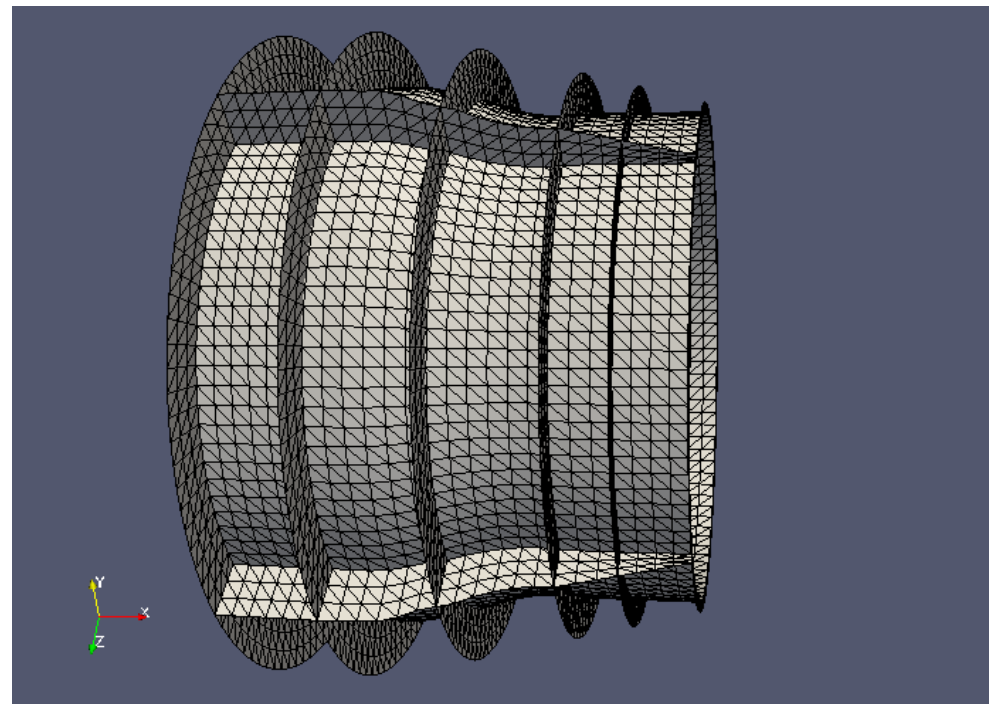

Fig. 2 Shape-parametric jet engine nozzle.

\section{B. Model-form uncertainties and what-if? scenarios associated with shape changes}

The first shape-parametric FE HDM described in Section V|A is equipped with an accurate representation of the variable thickness of the nozzle also described in that section. In the absence of true experimental data, this shapeparametric HDM model is used as a surrogate experimental system. On the other hand, the second shape-parametric FE HDM described in Section V|A from which all PROMs, HPROMs and SHPROMs are derived, is equipped with a constant representation of the thickness set to $6.97 \times 10^{-4} \mathrm{~m}$ in order to simulate a model-form uncertainty. Otherwise, both shape-parametric FE HDMs are varied in the shape-parameter domain $\Omega_{\varrho}=(l, r) \in[0.9,1.1] \mathrm{m} \times[0.9,1.1] \mathrm{m}$. Hence, the main length of the nozzle and the radius of its circular cross section at any point along its axisymmetry axis is varied in the following what-if? vibration analysis scenarios by $\pm 10 \%$.

In all considered scenarios, the QoIs are set to the first eight natural frequencies of the nozzle. Their surrogate experimental values and the counterpart values predicted using the FE HDM at the four corners and at the center of $\Omega_{\varrho}$ are reported in Table 1, together with the relative discrepancies between these two sets of values of the QoIs. These discrepancies are reported to range between $0.05 \%$ and $5.23 \%$.

\section{Shape-parametric HPROM}

A global shape-parametric HPROM of the jet engine nozzle is first constructed using: the (second) shapeparametric HDM described above; the greedy sampling algorithm in the shape-parameter domain $\Omega_{\varrho}=(l, r) \in$ 
Table 1 Natural frequencies (in $\mathrm{Hz}$ ) predicted using the surrogate experimental system and the deterministic FE HDM, and relative discrepancies.

\begin{tabular}{|c|c|c|c|c|c|c|c|c|}
\hline & \multicolumn{8}{|c|}{ Surrogate experimental values (in Hz). } \\
$(l, r)$ & $f_{1}$ & $f_{2}$ & $f_{3}$ & $f_{4}$ & $f_{5}$ & $f_{6}$ & $f_{7}$ & $f_{8}$ \\
\hline $0.9,0.9$ & 544.48 & 559.41 & 755.12 & 755.12 & 867.98 & 867.98 & 1056.97 & 1063.41 \\
$0.9,1.1$ & 512.64 & 526.59 & 713.64 & 713.64 & 755.60 & 755.60 & 824.79 & 836.51 \\
$1.0,1.0$ & 486.17 & 499.71 & 676.26 & 676.26 & 771.72 & 771.72 & 859.58 & 862.39 \\
$1.1,0.9$ & 461.63 & 476.99 & 629.13 & 629.13 & 783.77 & 783.77 & 852.47 & 856.18 \\
$1.1,1.1$ & 438.84 & 451.44 & 611.61 & 611.61 & 694.06 & 694.06 & 711.52 & 713.12 \\
\hline & \multicolumn{8}{|c|}{ Numerically predicted values (in Hz) using HDM. } \\
$(l, r)$ & $f_{1}$ & $f_{2}$ & $f_{3}$ & $f_{4}$ & $f_{5}$ & $f_{6}$ & $f_{7}$ & $f_{8}$ \\
\hline $0.9,0.9$ & 555.79 & 568.15 & 771.96 & 771.96 & 904.69 & 904.69 & 1058.09 & 1064.41 \\
$0.9,1.1$ & 522.90 & 534.46 & 728.60 & 728.60 & 778.85 & 778.85 & 827.95 & 839.22 \\
$1.0,1.0$ & 496.36 & 507.56 & 691.25 & 691.25 & 804.91 & 804.91 & 859.96 & 862.86 \\
$1.1,0.9$ & 471.87 & 485.09 & 643.55 & 643.55 & 824.65 & 824.65 & 853.07 & 856.84 \\
$1.1,1.1$ & 448.08 & 458.55 & 624.95 & 624.95 & 711.76 & 713.45 & 723.79 & 723.89 \\
\hline & \multicolumn{8}{|c|}{ Relative discrepancies $(\%)}$. \\
$(l, r)$ & $f_{1}$ & $f_{2}$ & $f_{3}$ & $f_{4}$ & $f_{5}$ & $f_{6}$ & $f_{7}$ & $f_{8}$ \\
\hline $0.9,0.9$ & -2.18 & -1.56 & -2.23 & -2.23 & -4.23 & -4.23 & -0.11 & -0.09 \\
$0.9,1.1$ & -2.00 & -1.49 & -2.10 & -2.10 & -3.08 & -3.08 & -0.38 & -0.32 \\
$1.0,1.0$ & -2.10 & -1.57 & -2.22 & -2.22 & -4.30 & -4.30 & -0.04 & -0.05 \\
$1.1,0.9$ & -2.22 & -1.70 & -2.29 & -2.29 & -5.22 & -5.22 & -0.07 & -0.09 \\
$1.1,1.1$ & -2.11 & -1.57 & -2.18 & -2.18 & -2.55 & -2.79 & -1.72 & -1.51 \\
\hline
\end{tabular}

$[0.9,1.1] \mathrm{m} \times[0.9,1.1] \mathrm{m}$ and an $11 \times 11$ uniform grid for pre-sampling candidate shape-parameter points at each iteration; and the ECSW hyperreduction method equipped with the non-negative LS optimization problem (IV.6) for determining the weight vector $\xi$ and the tolerance $\varepsilon=10^{-4}$. At each sampled shape-parameter point: the linear structural dynamics problem (II.2) is solved using $f^{e x t}(\varrho, t)=0$ and a broadband initial displacement $u_{0}$ and a corresponding broadband initial velocity $v_{0}$ that excite all 8 natural modes of interest; 500 solution snapshots are collected in the time-interval $\left[0,5 \times 10^{-3}\right] \mathrm{s}$ at the sampling time-step $\Delta s=1 \times 10^{-5} \mathrm{~s}$; and these snapshots are accumulated in the snapshot matrix $X$ which is compressed into a global deterministic ROB $V$ using a truncated SVD that captures $99.99 \%$ of the energy of the snapshots(see (II.3) ).

The greedy sampling algorithm is initialized using $\left(l_{1}, r_{1}\right)=(0.92,0.95)$ as the first sampled shape-parameter point. It is run until the calibrated error indicator falls below the value of 0.5. It samples a total of five shape-parameter points in $\Omega_{\varrho}$, and produces an HPROM of dimension $n=28$ that it computes on a reduced mesh $\widetilde{\mathcal{E}}$ with 364 elements and 920 nodes (see Fig. 3).

Fig. 4 shows the progression of the indicated error on the aforementioned $11 \times 11$ grid during the five iterations performed by this algorithm. In this figure, the black-filled bullets designate the shape-parameter points sampled by the greedy sampling algorithm at the previous iteration in order to update the global HPROM, and each red-filled bullet designates the shape-parameter point sampled at the end of the current iteration.

Given that for this application the (second) shape-parametric FE HDM has a small size, the true HPROM errors are computed at each iteration of the greedy sampling algorithm in order to verify the performance of the proposed error indicator (II.8). Fig. 5, which graphically depicts the variations on the aforementioned $11 \times 11$ uniform grid of both of the indicated and true HPROM errors at the last iteration performed by the greedy sampling algorithm, confirms the suitablity of the proposed error indicator. The final error (which is the sum of the true relative HPROM errors for all QoIs) at any of the sampled shape-parameter points is less than 0.34 . The true relative HPROM error for any of the QoIs at any of the sampled shape-parameter points is less than $8 \%$. 


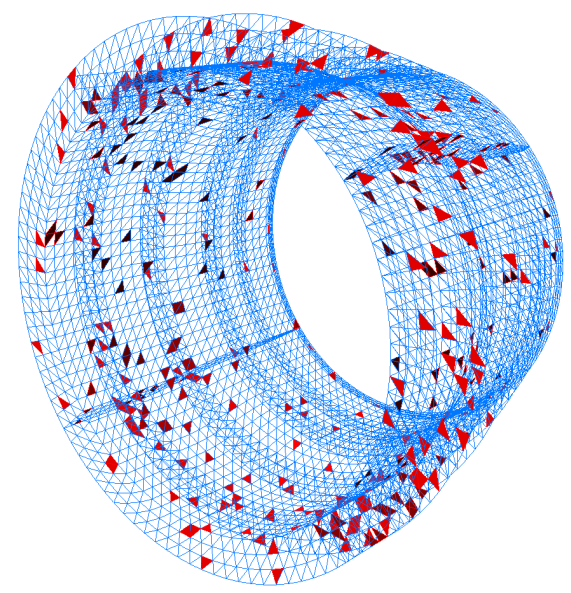

Fig. 3 Reduced mesh constructed by the ECSW hyperreduction method (sampled elements are highlighted in red color).

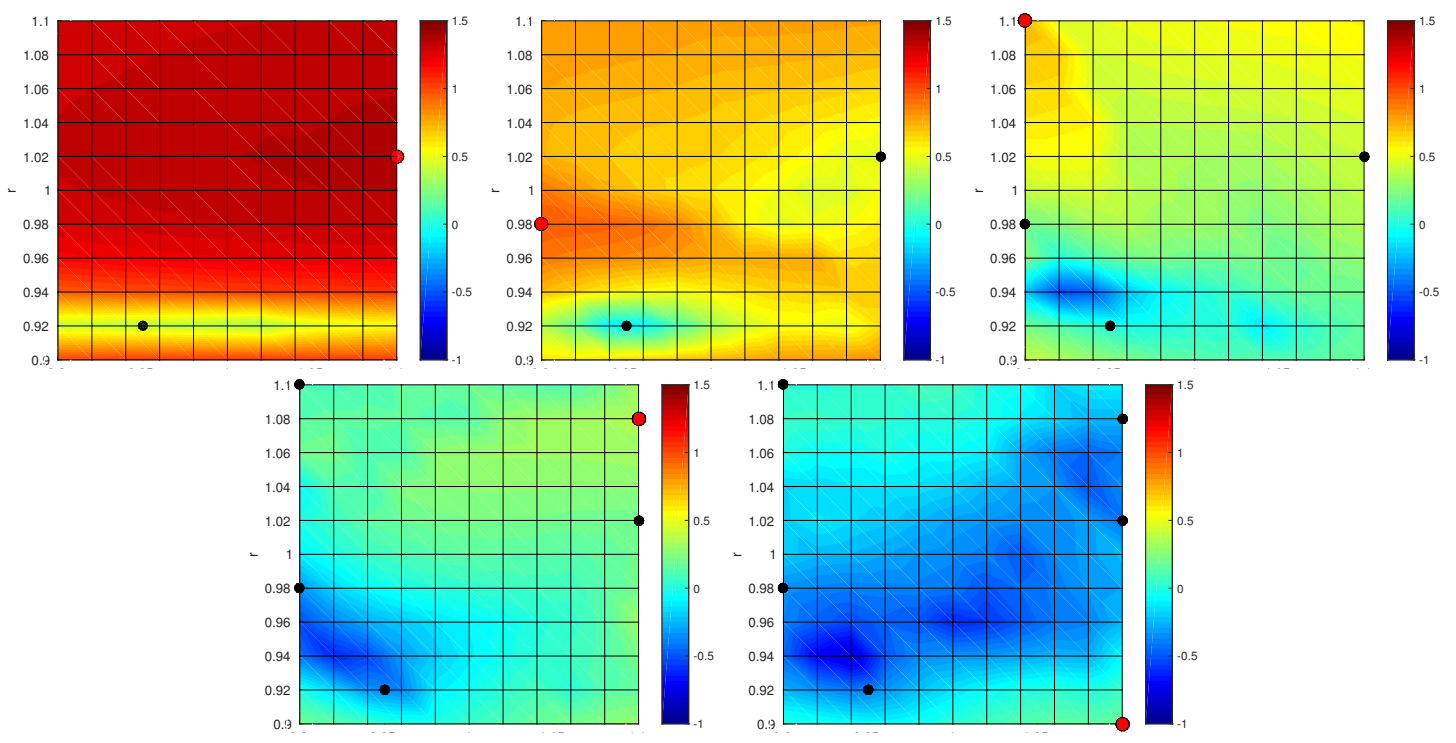

Fig. 4 Progression of the indicated error $\left(\log _{10}\right)$ on an $11 \times 11(l, r)$ grid during the iterations performed by the greedy sampling algorithm.
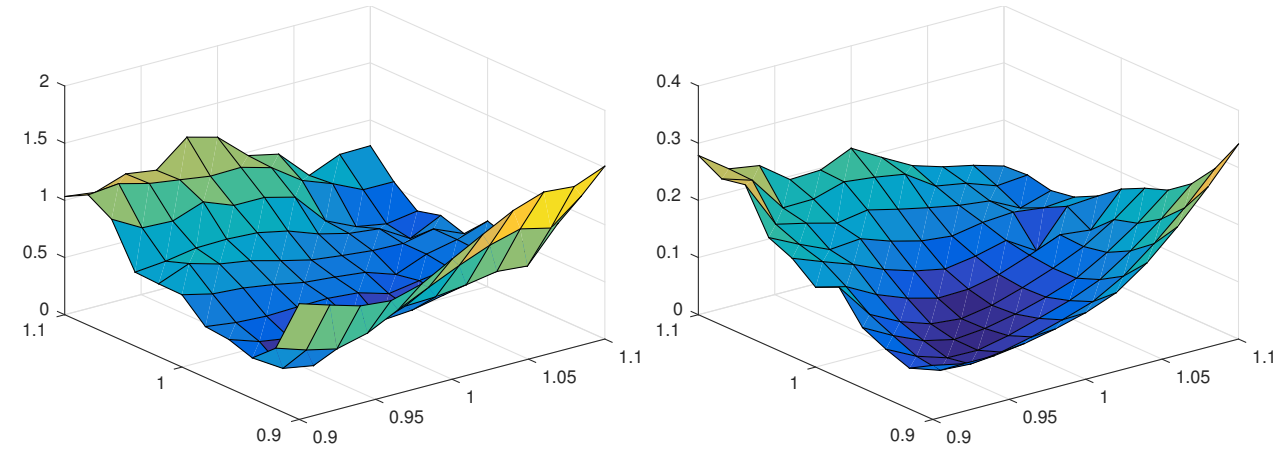

Fig. 5 Variations on an $11 \times 11(l, r$ grid of the indicated (left) and true (right) HPROM errors upon declared convergence of the greedy sampling algorithm. 


\section{Shape-parametric SHPROM}

Following the construction of the global HPROM of dimension $n=28$ described above, an SROB V and associated SHPROM of the same dimension are constructed on the computed reduced mesh using the procedures outlined in Section III and Section IV] To identify the hyperparameter vector, the decoupled minimization problem [IV.11] is solved using the weight vector computed by ECSW for the global HPROM and the following settings:

- $n_{\varrho}=5, \varrho_{1}=(0.9,0.9), \varrho_{2}=(1.1,0.9), \varrho_{3}=(0.9,1.1), \varrho_{4}=(1.1,1.1), \varrho_{5}=(1.0,1.0)$, and $m_{u}=m_{o}=8$.

- $o^{\text {ref }}=o^{\text {exp }}$, and $o^{\text {exp }}=\left(f_{1}^{\text {exp }}, f_{2}^{\text {exp }}, f_{3}^{\text {exp }}, \cdots, f_{m_{o}}^{\text {exp }}\right)$, where $f_{j}^{\text {exp }}, j=1, \cdots, m_{o}$, denotes the $j$-th natural frequency computed using the surrogate experimental system (first FE HDM).

- $v_{j}^{\text {ref }}, j=1, \cdots, m_{o}$, defined as follows

$$
v_{j}^{\text {ref }}=\gamma\left|o_{j}^{\text {ref }}-o_{j}\right|, \quad j=1, \ldots m_{o}
$$

where $o_{j}=\varpi_{j} / 2 \pi, j=1, \cdots, m_{o}$, and $\gamma$ is set to $\gamma=1.2$.

The weight $w_{J}$ is set to 0.95 so that the target for $J_{\text {mean }}$ is preponderant during the identification of the hyperparameter vector $\alpha$. The solution of the second minimization problem in (IV.11) is computed using the MATLAB function fmincon, its active-set algorithm, and the following constraints:

$$
\begin{array}{r}
10^{-5} \leq s \leq 10^{-2} \\
0.001 \leq \beta \leq 0.1 \\
0 \leq \tau_{j j} \leq 20,1 \leq j \leq n \quad \text { and } \quad-10 \leq \tau_{i j} \leq 10,1 \leq i<j \leq n \\
0.9999 \leq s_{M} \leq 2.0
\end{array}
$$

The chosen optimization algorithm is initialized with $\alpha_{0}=\left(s_{0}, \beta_{0}, s_{M_{0}}, \tau_{0}\right)$, where $s_{0}=4 \times 10^{-5}, \beta_{0}=4 \times 10^{-2}$, $s_{M_{0}}=1$, and $\tau_{0}=I_{n}$. The probability distributions of the random variables $\mathbf{s}, \boldsymbol{\beta}$, and $\mathbf{s}_{M}$ are chosen as uniform distributions on the supports defined by their constraints. The probability model of the random matrix $\tau$ is constructed as follows. For $1 \leq i<j \leq n, \tau_{i j}=\tau_{0 i j}\left(1+\mathcal{N}_{i j}\right)$, and for $i=1, \ldots, n, \tau_{i i}=\tau_{0 i i}\left|0.728+\mathcal{N}_{i i}\right|$, where $\left\{\mathcal{N}_{i j}\right\}_{1 \leq i \leq j \leq n}$ is a family of $n(n+1) / 2$ independent variables. Each of these variables is a Gaussian, centered variable with a unit variance. Note that the absolute value is introduced in the expression of $\tau_{i i}$ in order to guarantee the required positiveness of the diagonal terms of the matrix $\tau$.

The Monte Carlo method is used as a stochastic solver for estimating the cost function $J(\alpha)$ (III.11) for every value of the hyperparameter vector $\alpha$ proposed by the optimizer. Specifically, 10 independent realizations of the QoIs are performed for each estimation of $J(\alpha)$. After 250 iterations, the value of this cost function is $3.65 \cdot 10^{-3}$ : it is reached for $\alpha=\left(s, s_{M}, \beta, \sigma\right)^{T}$ where $s=1.4182 \times 10^{-5}, s_{M}=1.2391, \beta=8.7882 \cdot 10^{-2}$, and the 406 entries of $\tau$ are not reported for obvious reasons.

\section{E. Sample CPU performance}

As stated earlier, the main purpose of the SHPROM it to ensure the computational feasibility of the nonparametric probabilistic method described in this paper for performing model-form UQ. Its construction, which is performed here in MATLAB, requires thousands of evaluations of the cost function $J(\alpha)$ (III.11). Even though many of these evaluations can be executed in parallel, a single such evaluation is indicative of the overall CPU requirement. Hence, in order to illustrate the CPU performance of the SHPROM constructed for the application described herein, and the performance improvement this SHPROM delivers over the SPROM, the CPU cost for a single cost function evaluation is reported in Table 2 for each of the two stochastic reduced-order models. Each reported CPU time accounts for building the SPROM/SHPROM and performing 10 generated realizations - that is, solving the stochastic reduced/hyperreduced generalized EVP - at each of the five shape-parameter points sampled by the greedy sampling procedure when building the global ROB $V$ (which implies the solution of 50 reduced-order generalized EVPs).

The results reported in Table 2 show that for this application where the HDM has a small dimension $(N=27,240)$, hyperreduction accelerates the construction of the reduced-order model by a factor larger than 8 , and reduces the total CPU time by factor 5 .

\section{F. Modeling and quantification of model-form uncertainties}

The SHPROM whose construction is described in Section V|D is applied here to perform the model-form UQ analysis of the (second) HDM, while accounting for all modeling errors introduced by model reduction. Sample results 
Table 2 Performance comparisons of the SPROM and SHPROM for a single cost function evaluation (solution of 50 reduced-order generalized EVPs).

\begin{tabular}{|c|c|c|c|}
\hline Computational model & $\begin{array}{c}\text { CPU time elapsed } \\
\text { in model building }\end{array}$ & $\begin{array}{c}\text { CPU time elapsed in solution of } \\
\text { 50 reduced-order generalized EVPs }\end{array}$ & Total elapsed CPU time \\
\hline SPROM & $1.414 \mathrm{~s}$ & $0.021 \mathrm{~s}$ & $2.9717 \mathrm{~s}$ \\
\hline SHPROM & $0.171 \mathrm{~s}$ & $0.021 \mathrm{~s}$ & $0.5997 \mathrm{~s}$ \\
\hline
\end{tabular}

of this analysis are reported in Fig. 6 and Fig. 7 for two shape-parameter points in the shape-parameter domain: the lower left corner $(0.9,0.9)$ of $\Omega_{\varrho}$, and the upper right corner $(1.1,1.1)$ of $\Omega_{\varrho}$. Mean values and confidence intervals corresponding to the quantiles $98 \%$ and $2 \%$ are constructed using 100 QoI realizations generated by the SHPROM.

The reader can observe in both figures that the global HPROM constructed for this problem reproduces the data generated by the HDM accurately. This is due to the greedy sampling procedure and its performance discussed in Section $V \mid C$ The reader can also observe that: the surrogate experimental data is captured reasonably well by the mean values obtained using the SHPROM; and the realizations generated using this SHPROM and the resulting confidence interval corresponding to the quantiles $98 \%$ and $2 \%$ encompass the deterministic value of the surrogate experimental value of each QoI.

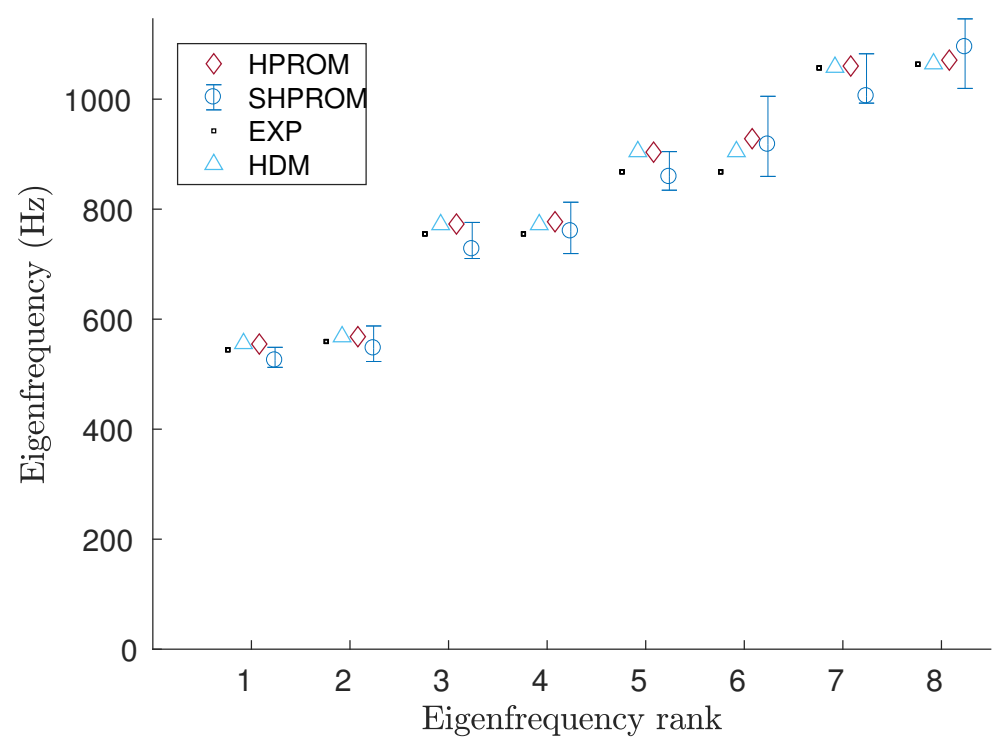

Fig. 6 Vibration analysis at $(l, r)=(0.9,0.9)$ : QoIs (natural frequencies) predicted using the deterministic HPROM; mean values and confidence intervals corresponding to the quantiles $98 \%$ and $2 \%$ constructed using the SHPROM; surrogate experimental values (EXP) of the QoIs; and QoIs predicted using the HDM.

\section{Acknowledgments}

All U.S. authors acknowledge partial support by the Army Research Laboratory through the Army High Performance Computing Research Center under Cooperative Agreement W911NF-07-2-0027, and partial support by DARPA under the Enabling Quantification of Uncertainty in Physical Systems (EQUiPS) program.

[1] https://en.wikipedia.org/wiki/Uncertainty_quantification.

[2] Soize C. A nonparametric model of random uncertainties for reduced matrix models in structural dynamics. Probabilistic Engineering Mechanics 2000; 15(3):277-294. 


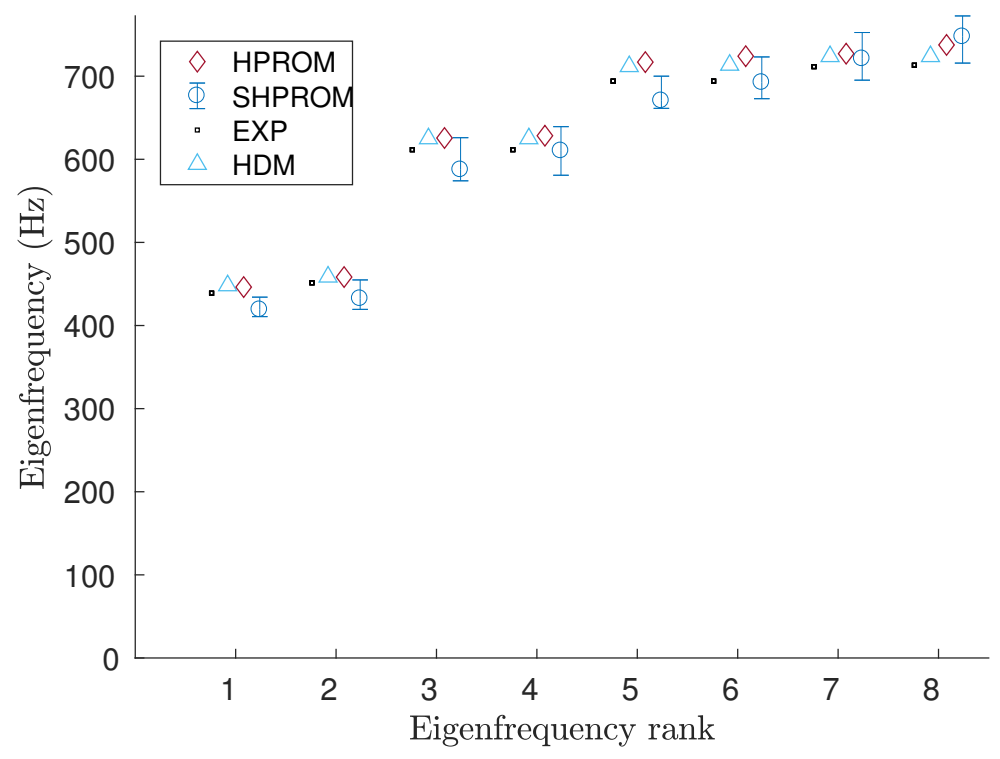

Fig. 7 Vibration analysis at $(l, r)=(1.1,1.1)$ : QoIs (natural frequencies) predicted using the deterministic HPROM; mean values and confidence intervals corresponding to the quantiles $98 \%$ and $2 \%$ constructed using the SHPROM; surrogate experimental values (EXP) of the QoIs; and QoIs predicted using the HDM.

[3] Soize C. Random matrix theory for modeling random uncertainties in computational mechanics. Computer Methods in Applied Mechanics and Engineering 2005; 194(12-16):1333-1366.

[4] Mignolet MP, Soize C. Stochastic reduced order models for uncertain nonlinear dynamical systems. Computer Methods in Applied Mechanics and Engineering 2008; 197(45-48):3951-3963.

[5] Soize C, Farhat C. A nonparametric probabilistic approach for quantifying uncertainties in low- and high-dimensional nonlinear models. International Journal for Numerical Methods in Engineering 2017; 109 (6):837-888.

[6] Farhat C, Bos A, Avery P, Soize C. Modeling and quantification of model-form uncertainties in eigenvalue computations using a stochastic reduced-order model. AIAA Journal 2017; DOI: 10.2514/1.J056314.

[7] Sirovich L. Turbulence and the dynamics of coherent structures. I-Coherent structures. II-Symmetries and transformations. III-Dynamics and scaling. Quarterly of Applied Mathematics 1987; 45:561-571.

[8] Farhat C, Avery P, Chapman T, Cortial J. Dimensional reduction of nonlinear finite element dynamic models with finite rotations and energy-based mesh sampling and weighting for computational efficiency. International Journal for Numerical Methods in Engineering 2014; 98(9):625-662.

[9] Veroy K, Patera A.-T. Certified real-time solution of the parametrized steady incompressible Navier-Stokes equations: rigorous reduced-basis a posteriori error bounds. International Journal for Numerical Methods in Fluids 2005; 47:773-788.

[10] Bui-Thanh T, Willcox K, Ghattas O. Parametric reduced-order models for probabilistic analysis of unsteady aerodynamic applications. AIAA Journal 2008; 46:2520-2529.

[11] Farhat C, Chapman T, Avery P. Structure-preserving, stability, and accuracy properties of the energy-conserving sampling and weighting (ECSW) method for the hyper reduction of nonlinear finite element dynamic models. International Journal for Numerical Methods in Engineering 2015; 102:1077-1110.

[12] Chapman T, Avery P, Collins JP, Farhat C. Accelerated mesh sampling for the hyper reduction of nonlinear computational methods. International Journal for Numerical Methods in Engineering 2017; 109:1623-1654.

[13] Farhat C, Geuzaine P, Brown G. Application of a three-field nonlinear fluid-structure formulation to the prediction of the aeroelastic parameters of an F-16 fighter. Computers \& Fluids 2003; 32:3-29.

[14] Geuzaine P, Brown G, Harris C, Farhat C. Aeroelastic dynamic analysis of a full F-16 configuration for various flight conditions. AIAA Journal 2003; 41:363-371. 